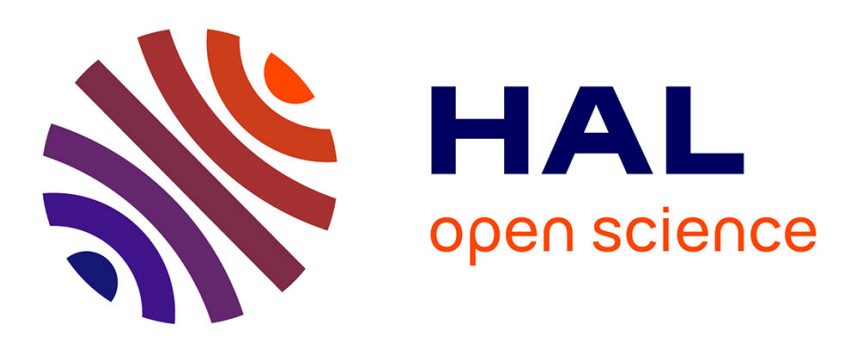

\title{
Hierarchical Robust Performance Analysis of Uncertain Large Scale Systems
}

Khaled Laib, Anton Korniienko, Marc Dinh, Gérard Scorletti, Florent Morel

\section{To cite this version:}

Khaled Laib, Anton Korniienko, Marc Dinh, Gérard Scorletti, Florent Morel. Hierarchical Robust Performance Analysis of Uncertain Large Scale Systems. IEEE Transactions on Automatic Control, 2018, 63 (7), pp.2075-2090. 10.1109/TAC.2017.2762468 . hal-01616980

\section{HAL Id: hal-01616980 \\ https://hal.science/hal-01616980}

Submitted on 15 Oct 2017

HAL is a multi-disciplinary open access archive for the deposit and dissemination of scientific research documents, whether they are published or not. The documents may come from teaching and research institutions in France or abroad, or from public or private research centers.
L'archive ouverte pluridisciplinaire HAL, est destinée au dépôt et à la diffusion de documents scientifiques de niveau recherche, publiés ou non, émanant des établissements d'enseignement et de recherche français ou étrangers, des laboratoires publics ou privés. 


\title{
Hierarchical robust performance analysis of uncertain large scale systems
}

\author{
Khaled Laib, Anton Korniienko, Marc Dinh, Gérard Scorletti and Florent Morel
}

\begin{abstract}
In this paper, the problem of robust performance analysis of interconnected uncertain systems with hierarchical structure is invstigated. The computational load associated to such problems does not allow a direct application of robustness analysis usual tools. To overcome this difficulty, we exploit the hierarchical structure of the problem and propose an algorithm to perform robustness analysis using IQC "propagatio" along the hierarchical structure. This algorithm allows to establish a tradeoff between computation time required to perform the analysis and the conservatism of the obtained results. Furthermore, it is possible to perform parallel computation using the proposed algorithm.
\end{abstract}

Index Terms-Uncertain large scale systems, robustness analysis, IQC analysis, LMI optimization, hierarchical approach, IQC propagation.

\section{INTRODUCTION}

$\mathrm{R}$ Obustness analysis of uncertain large scale systems (LSS) to ensure a certain level of performance in the worst case scenario is a major topic in the automatic control community. These LSS (networks) are obtained by interconnecting smaller subsystems within the objective of ensuring some global tasks. Therefore, it is natural to consider the hierarchical structure of networks: subsystems define the local level while their interconnection define the global level. In this paper, we are interested in robustness analysis of uncertain large scale systems with hierarchical structure.

Robustness analysis of uncertain LSS is a problem with many challenges. Major difficulties that frequently arise in this problem are: robustness analysis and large scale aspects. Although the robustness analysis is an NP hard problem [1], many efficient methods have been developed based on relaxations as convex optimization problem under Linear Matrix Inequality (LMI) constraints [2], see e.g. the $\mu$-upper bound [3] in the $\mu$-analysis approach [4] or the Integral Quadratic Constraint (IQC) approach [5].

The second aspect is the large scale associated to networks. Even when we consider the interconnection of systems without any uncertainties, the analysis problem remains complicated and the network stability is not easy to certify. In this case, the

Khaled Laib, Anton Kornnienko, Gérard Scorletti and Florent Morel are with Laboratoire Ampère Dpt. EEA of the Ecole Centrale de Lyon, Université de Lyon, 69134 Ecully Cedex, France: khaled.laibedoctorant.ec-lyon.fr, <anton.korniienko>, <gerard.scorletti>,

$<$ florent.morel $>$ dec-lyon.fr.

Marc Dinh is with MaIAGE, INRA, Université Paris-Saclay, 78350 Jouy-en-Josas, France: marc.dinh@jouy.inra.fr. robustness of the LSS is discussed with respect to the network size and its interconnection topology.

The objective is to establish decentralized conditions to ensure the stability of the LSS i.e. conditions that subsystems have to satisfy with respect to their interconnection matrix to guarantee the overall stability of the network. These conditions are obtained in different frameworks: [6] and [7] for dissipativity approaches, [8] and [9] for $S$-hull convexification approaches, [10] and [11] for graph theory approaches, etc.. Nevertheless, when considering both aspects (robustness and large scale), the complexity and the computation time dramatically increase due to the large size optimization problem we have to solve. Therefore, the robustness analysis usual tools cannot be practically applied directly.

In order to reduce this computational load, researchers focused on exploiting the particular characteristics of the subsystems and their interconnections. The authors of [12] propose a decomposable approach to investigate the robustness of uncertain LSS. The obtained conditions involve the structured singular values of the individual systems and the eigenvalues of the interconnection matrix. However, these results are valid only when the subsystems are homogeneous. Within the framework of IQC, characterizations of local systems and/or interconnections are used to obtain robust stability conditions in the case where the interconnection matrix is normal [13], unitarily diagonalized [14] or sparse with a chordal pattern [15] and [16].

However for a given LSS, it could be difficult to model the network with a normal, unitarily diagonalized or interconnection matrix with a chordal pattern. Furthermore, even if the network presents one of these structures, the previous methods allow only to investigate the stability. Introducing extra signals to investigate the performance may change the structure. In addition, these results do not exploit an important aspect of the problem which is the hierarchical structure. In this context, the Hierarchical approach was initially introduced in [17], in the case of conic uncertainty i.e. non structured dynamical uncertainty, to split the overall analysis problem into several low dimensional ones. Each uncertain system in the network can be characterized with conic properties which are a special case of IQC. The overall analysis is then performed in a hierarchical manner by propagating conic properties along the hierarchical structure. Nevertheless, this approach was not really exploited and implemented because it has neither been explained nor formulated how to obtain these IQC characterizing uncertain systems. 
In this paper, we adapt the hierarchical approach and extend it to the case of structured uncertainties. As a first contribution of this paper, we define a set of IQC characterizing different informations on the uncertain system: gain uncertainty, phase uncertainty, mixed gain-phase uncertainty. The IQC set thus defines a basis to characterize uncertain systems. In order to reduce the conservatism of this approach, a size measure is defined and minimized for each of the basis element. The problem of minimizing each size measure is formulated as an LMI optimization problem which can be solved efficiently. A second contribution of this paper is an algorithm to investigate the robust performance of the overall uncertain large scale system using basis propagation along the hierarchical structure. This algorithm allows to establish a trade-off between computation time required for the analysis and the conservatism of the results. Preliminary results can be found in [18]-[20].

\section{Paper outline}

This paper is organized as follows: Section II presents the formulation of uncertain LSS performance analysis problem. Section III presents the usual approach to perform robustness analysis of uncertain systems using dissipativity properties. Section IV presents the proposed approach to solve the problem of robustness analysis of uncertain LSS using basis propagation. The formulation and practical computation of the basis elements are presented in Section V. The benefits of the hierarchical approach, compared to the direct analysis method, are illustrated through two application examples in Section VI. We discuss in Section VII the algorithmic complexity and the computation time required to perform robustness analysis for a sub-class of uncertain LSS with hierarchical structure. Conclusions and perspectives are presented in Section VIII.

\section{Notations}

$\mathbb{R}$ and $\mathbb{C}$ are the sets of real and complex numbers respectively. $\overline{\mathbb{R}}^{+}=\mathbb{R}^{+} \cup\{+\infty\}$ where $\mathbb{R}^{+}$is the set of real positive numbers including $0 . \overline{\mathbb{C}}^{+}$denotes the closed right half of complex plane including imaginary axis and infinity. The maximum singular value of a matrix $M$ is denoted $\bar{\sigma}(M)$. If the index of a matrix or a signal is not relevant and can be understood from the context, then this index will be omitted or replaced with "•". The real and imaginary parts of the complex entity $\bullet$ are denoted $\operatorname{Re}(\bullet)$ and $\operatorname{Im}(\bullet)$ respectively. $\mathbf{R H}_{\infty}$ (respectively $\mathbf{R L}_{\infty}$ ) denotes the set of matrices of stable (resp. non causally stable) rational transfer functions.

We consistently denote elementary uncertainties by $\Delta$ and interconnections by $M$ which can be partitioned into $M=\left(\begin{array}{ll}M_{11} & M_{12} \\ M_{21} & M_{22}\end{array}\right)$. For several matrices $M_{j}$, $\operatorname{bdiag}_{j}\left(M_{j}\right)$ denotes the block diagonal matrix composed of $M_{j}$. We denote by $\boldsymbol{\Delta} \star M$ the set $\{\Delta \star M, \forall \Delta \in \boldsymbol{\Delta}\}$, referred to as an uncertain system, defined by $\Delta \star M=M_{22}+$ $M_{21} \Delta\left(I-M_{11} \Delta\right)^{-1} M_{12}$ with $\star$ standing for the Redheffer star product and it will be referred to as the Linear Fractional Transformation (LFT) interconnection of $M$ and $\Delta$.
Finally, we denote by $\mathcal{L}_{\mathcal{S}}\left(\bullet, \Phi_{11}, \Phi_{12}, \Phi_{22}\right)$ the matrix

$$
\left(\begin{array}{c}
\bullet \\
I
\end{array}\right)^{*}\left(\begin{array}{l|l}
-\Phi_{22} & -\Phi_{12}^{*} \\
\hline-\Phi_{12} & -\Phi_{11}
\end{array}\right)\left(\begin{array}{c}
\bullet \\
I
\end{array}\right)
$$

and we denote by $\mathcal{L}_{\mathcal{P}}\left(\bullet, \Phi_{11}, \Phi_{12}, \Phi_{22}, X, Y, Z, \epsilon\right)$ the matrix

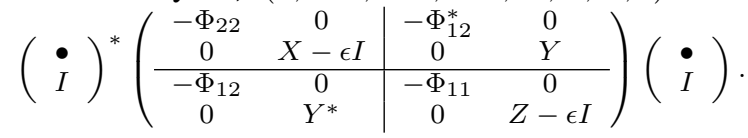

\section{Problem Formulation}

\section{A. LSS Hierarchical Structure}

Inspired by [17] and [18], an uncertain large scale system is defined by a tree composed of leaves interconnected through branches. These leaves and branches will be organized by levels. An example of tree is illustrated in Fig. 1 where a hierarchical structure arises with $l=4$ levels.



Fig. 1: Uncertain linear large scale system $T_{1}^{1}$ with hierarchical structure of four levels

An index $i$ is associated to each level i.e. $i=1, \ldots, l$. At each level $i$, two types of components may be found: leaves and branches.

The leaves represent the elementary uncertain components and they are denoted $\Delta_{j_{\Delta}}^{i} \in \Delta$ with $j_{\Delta} \in\left\{1, \ldots, N_{\Delta}^{i}\right\}$ where $N_{\Delta}^{i}$ is the number of elementary uncertain components at level $i$ and $\Delta$ is the uncertainty set traditionally considered in robust analysis literature. It is given as a block diagonal combination of elementary uncertainties

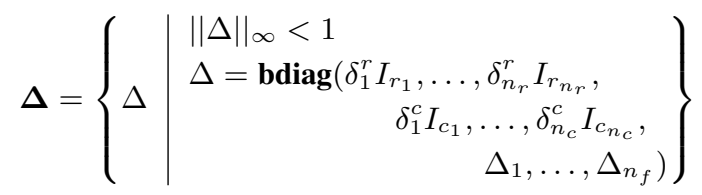

where

- $\delta_{j}^{r} \in \mathbb{R}$ is a real parametric uncertainty,

- $\delta_{j}^{c} \in \mathbb{C}$ is a complex uncertainty,

- $\Delta_{j}$ is a LTI system which represents a dynamical uncertainty with $k_{m}^{j}$ inputs and $k_{l}^{j}$ outputs. 
The input output signals of $\Delta_{j_{\Delta}}^{i}$ are $q_{j_{\Delta}}^{i}$ and $p_{j_{\Delta}}^{i}$ respectively. The elementary uncertain components $\Delta_{j_{\Delta}}^{i}$, of level $i$, are the end of the tree i.e. leaves since they are only connected to the certain components of the level $i-1$.

The branches are certain components denoted $M_{j_{M}}^{i}$, assumed to be LTI systems, with $j_{M} \in\left\{1, \ldots, N_{M}^{i}\right\}$ where $N_{M}^{i}$ is the number of certain components at the level $i$. In contrast with $\Delta_{j_{\Delta}}^{i}$, which are only connected to certain components of the level below, $M_{j_{M}}^{i}$ are connected to both levels: below and above. The certain components $M_{j_{M}}^{i}$ are connected to certain and to uncertain components from level $i+1$ and to certain components from level $i-1$. The signals $w_{j_{M}}^{i}$ and $p_{\bullet}^{i+1}$ (and possibly $z_{\bullet}^{i+1}$ ) are the input signals of $M_{j_{M}}^{i}$ while $z_{j_{M}}^{i}$ and $q_{\bullet}^{i+1}$ (and possibly $w_{\bullet}^{i+1}$ ) are the output signals.

After showing the different levels of the LSS with its different certain and uncertain components (which can be seen as an horizontal decomposition), it is possible to regroup the components connected vertically. The result will be an uncertain system denoted $T_{j}^{i}$, where $i$ stands for the hierarchical level and $j$ for the index of the uncertain system at this hierarchical level. The signals $w_{j}^{i}$ and $z_{j}^{i}$ are the input and output signals of the uncertain system $T_{j}^{i}$. The number of uncertain systems at each level $i$ is $N_{T}^{i}=N_{M}^{i}$.

Each uncertain system $T_{j}^{i}$ is the LFT interconnection of $M_{j}^{i}$ with either just only elementary uncertain components of the next hierarchical level $\left(\Delta_{\bullet}^{i+1}\right.$ for example) or a block diagonal composition of elementary uncertain components $\Delta_{\bullet}^{i+1}$ and uncertain systems $T_{\bullet}^{i+1}$ from the next hierarchical level.

For the ease of notation, and for the purpose of this paper, both cases will be combined into block diagonal augmented uncertainty that will be denoted $\Omega_{\bullet}^{i+1} \in \Omega$ where $\Omega$ is the extension of the elementary uncertainty set (1), that is

$$
\boldsymbol{\Omega}=\left\{\Omega \mid \Omega=\underset{j}{\operatorname{bdiag}}\left(\Omega_{j}\right)\right\}
$$

where $\Omega_{j}$ is either an elementary uncertain block i.e. $\Omega_{j} \in \Delta$, or an uncertain LTI system that belongs to a bounded and connected set without a priori "known" bound.

It is now possible to model the LSS as

$$
\begin{aligned}
& \forall i \in\{1, \ldots, l-1\} \\
& \forall j \in\left\{1, \ldots, N_{T}^{i}\right\} \\
& z_{j}^{i}=\underbrace{\Omega_{j}^{i+1} \star M_{j}^{i}}_{T_{j}^{i}} w_{j}^{i}
\end{aligned}
$$

with

$$
\Omega_{j}^{i+1}=\operatorname{bdiag}\left(\underset{m \in \mathcal{N}_{\Delta}\left(M_{j}^{i}\right)}{\operatorname{bdiag}}\left(\Delta_{m}^{i+1}\right) \underset{n \in \mathcal{N}_{T}\left(M_{j}^{i}\right)}{\operatorname{bdiag}}\left(T_{n}^{i+1}\right)\right)
$$

where $\mathcal{N}_{T}\left(M_{j}^{i}\right)$ is the index set of the uncertain systems $T_{\bullet}^{i+1}$ connected to $M_{j}^{i}$ and $\mathcal{N}_{\Delta}\left(M_{j}^{i}\right)$ is the index set of the elementary uncertain components $\Delta_{\bullet}^{i+1}$ connected to $M_{j}^{i}$ respectively.
For illustration purposes, let us consider the LSS presented in Fig. 1 where $w_{1}$ and $z_{1}$ are the input and the output signals respectively. They define the system $T_{1}^{1}$ for which we want to investigate the performance as it will be formally defined later. At level 2, the components can be regrouped into $n+1$ uncertain systems $T_{j}^{2}$, for $\forall j \in\{1, \ldots, n+1\}$, interconnected through $M_{1}^{1}$ to form $T_{1}^{1}$. Each $T_{j}^{2}$ is the LFT interconnection of $M_{j}^{2}$ with $\Omega_{j}^{3}$. Two types of $\Omega_{j}^{3}$ appear: either $\Omega_{j}^{3}=\Delta_{j}^{3}$ for $j=\{1, \ldots, n\}$ or $\Omega_{n+1}^{3}=\operatorname{bdiag}\left(\Delta_{n+1}^{3}, T_{1}^{3}\right)$. This last uncertain system $T_{1}^{3}$, used to construct $\Omega_{n+1}^{3}$, is in itself the interconnection of $M_{1}^{3}$ with $\Omega_{1}^{4}$ which is the block diagonal combination of two elementary uncertain components: $\Omega_{1}^{4}=\operatorname{bdiag}\left(\Delta_{1}^{4}, \Delta_{2}^{4}\right)$.

\section{B. Problem statement}

The LTI system performance is achieved if the maximum singular value $\bar{\sigma}(\bullet)$ of the system frequency response respects, along the frequencies, some user-defined frequency dependent constraints (see [21]). Since the uncertainties will impact the system frequency response, we denote $\gamma_{\omega}$ as an upper bound on the system maximum singular value $\bar{\sigma}(\bullet)$ over all the uncertainties along the frequencies. Hence, the robust performance analysis boils down to check if the computed minimal value $\gamma_{\omega}$, for each frequency $\omega \in \overline{\mathbf{R}}^{+}$, is less than the user defined bound for all possible uncertainties.

Now, the robust performance analysis problem of uncertain LSS can be formulated.

Problem 2.1 (Robust Performance): Given an uncertain LSS defined by (3) and (4) with $\Delta_{j}^{i} \in \boldsymbol{\Delta}, M_{j}^{i} \in \mathbf{R} \mathbf{H}_{\infty}$. Given $l, N_{T}^{i}, \mathcal{N}_{T}\left(M_{j}^{i}\right)$ and $\mathcal{N}_{\Delta}\left(M_{j}^{i}\right)$, test if the LSS is stable $\forall \Delta_{j}^{i} \in \boldsymbol{\Delta}$ for all $i, j$. Furthermore, for every frequency $\omega \in \overline{\mathbf{R}}^{+}$solve

$$
\begin{array}{cl}
\min _{\gamma_{\omega}} & \gamma_{\omega} \\
\text { subject to } & \bar{\sigma}\left(T_{1}^{1}(\mathbf{j} \omega)\right)<\gamma_{\omega} \quad \forall \Delta_{j}^{i} \in \boldsymbol{\Delta}
\end{array}
$$

\section{Road-map}

Problem 2.1 is the main problem under consideration in this paper and it may be solved using robustness analysis usual tools which will be presented in Section III if the LSS size is not important. However, when the LSS size becomes important, the efficient resolution of Problem 2.1 is not possible using these tools due to the large size of the associated optimization problem and the dramatic increase of computation time. Algorithm 1 presented in Section IV allows to solve Problem 2.1 efficiently by exploiting the LSS hierarchical structure. Algorithm 1 allows to split the overall analysis problem into several low dimensional ones. In order to solve each of these problems, the input output signals of each uncertain subsystem in the LSS are characterized using and combining different relevant information such as gain uncertainty, phase uncertainty, etc. This characterization is presented in Section V and it represents a generalization of the robustness analysis usual tools presented in Section III. 
The gain uncertainty problem (Problem 5.1) is solved using Theorem 5.1, the phase uncertainty problem (Problem 5.3) is solved using Theorem 5.3 and the mixed gain/phase uncertainty problem (Problem 5.2) is solved using Theorem 5.2.

\section{RobUStNeSS ANALYSIS OF UnCERTAin Systems}

\section{A. Uncertain systems}

An uncertain system will be defined as an interconnection $T=\Omega \star M$ with $M \in \mathbf{R H}_{\infty}$ and $\Omega \in \Omega$ where $\boldsymbol{\Omega}$ is bounded and connected set of LTI systems as in (2). Introducing internal signals and using frequency domain, we obtain the following system description

$$
\begin{aligned}
p(\mathbf{j} \omega) & =\Omega(\mathbf{j} \omega) \\
\left(\begin{array}{l}
q(\mathbf{j} \omega) \\
z(\mathbf{j} \omega)
\end{array}\right) & =M(\mathbf{j} \omega)\left(\begin{array}{l}
p(\mathbf{j} \omega) \\
w(\mathbf{j} \omega)
\end{array}\right)
\end{aligned}
$$

where $w(\mathbf{j} \omega) \in \mathbb{C}^{n_{w}}$ and $z(\mathbf{j} \omega) \in \mathbb{C}^{n_{z}}$ are the input and the output signals respectively and they will be used to define and evaluate the system performance as it will be explained later. The signals $p(\mathbf{j} \omega) \in \mathbb{C}^{n_{p}}$ and $q(\mathbf{j} \omega) \in \mathbb{C}^{n_{q}}$ are internal signals.

\section{B. Robustness analysis}

We will use dissipativity properties to characterize the performance of an uncertain system $T$. In the field of robust control theory, IQC are often used to characterize the system behavior in form of input-output signal relations (also known as graph relations).

Definition 3.1 (IQC [5]): The two signals $w(\mathbf{j} \omega)$ and $z(\mathbf{j} \omega)$ are said to satisfy the IQC defined by $\Phi=\Phi^{*}$ of $\mathbf{R} \mathbf{L}_{\infty}$, if $\exists \epsilon>0$ and

$\int_{-\infty}^{+\infty}\left(\begin{array}{c}z(\mathbf{j} \omega) \\ w(\mathbf{j} \omega)\end{array}\right)^{*} \Phi(\mathbf{j} \omega)\left(\begin{array}{c}z(\mathbf{j} \omega) \\ w(\mathbf{j} \omega)\end{array}\right) d \omega \geq \epsilon \int_{-\infty}^{+\infty}\left(\begin{array}{c}z(\mathbf{j} \omega) \\ w(\mathbf{j} \omega)\end{array}\right)^{*}\left(\begin{array}{c}z(\mathbf{j} \omega) \\ w(\mathbf{j} \omega)\end{array}\right) d \omega$

Since the problem considered in this paper is the performance analysis of uncertain LTI systems i.e. $z(\mathbf{j} \omega)=T(\mathbf{j} \omega) w(\mathbf{j} \omega)$, the integral term could be dropped which gives a Quadratic Constraint (QC). Furthermore if

$$
\Phi(\mathbf{j} \omega)=\left(\begin{array}{cc}
X(\mathbf{j} \omega) & Y(\mathbf{j} \omega) \\
Y(\mathbf{j} \omega)^{*} & Z(\mathbf{j} \omega)
\end{array}\right)
$$

with $X=X^{*}, Z=Z^{*}$ and $Y$ are transfer functions of $\mathbf{R} \mathbf{L}_{\infty}$, the QC thus defines a dissipativity property and the uncertain system $\Omega \star M$ is said to be $\{X(\mathbf{j} \omega), Y(\mathbf{j} \omega), Z(\mathbf{j} \omega)\}$ dissipative if

$$
\begin{gathered}
\left(\begin{array}{c}
\Omega(\mathbf{j} \omega) \star M(\mathbf{j} \omega) \\
I
\end{array}\right)^{*}\left(\begin{array}{cc}
X(\mathbf{j} \omega) & Y(\mathbf{j} \omega) \\
Y(\mathbf{j} \omega)^{*} & Z(\mathbf{j} \omega)
\end{array}\right)\left(\begin{array}{c}
\Omega(\mathbf{j} \omega) \star M(\mathbf{j} \omega) \\
I
\end{array}\right) \\
\geq \epsilon\left(\begin{array}{c}
\Omega(\mathbf{j} \omega) \star M(\mathbf{j} \omega) \\
I
\end{array}\right)^{*}\left(\begin{array}{c}
\Omega(\mathbf{j} \omega) \star M(\mathbf{j} \omega) \\
I
\end{array}\right)
\end{gathered}
$$

Remark 3.1: The performance constraint of the LSS $T_{1}^{1}$ in Problem 2.1 can be expressed in terms of dissipativity properties as following $\bar{\sigma}\left(T_{1}^{1}(\mathbf{j} \omega)\right)<\gamma_{\omega}, \forall \Delta_{j}^{i} \in \boldsymbol{\Delta} \Longleftrightarrow T_{1}^{1}$ is $\left\{-I, 0, \gamma_{\omega}^{2} I\right\}$ dissipative $\forall \Delta_{j}^{i} \in \Delta$.

In order to keep the discussion as general as possible, we will use, in this paper, a general performance measure defined by a general $\{X(\mathbf{j} \omega), Y(\mathbf{j} \omega), Z(\mathbf{j} \omega)\}$ dissipativity.
The following theorem gives necessary and sufficient conditions to perform the robust performance analysis of an uncertain system $\Omega \star M$ for every $\Omega \in \Omega$

Theorem 3.1: Let $\Omega$ be a bounded and connected set of LTI systems. The uncertain system $\Omega \star M$ is stable and $\{X(\mathbf{j} \omega), Y(\mathbf{j} \omega), Z(\mathbf{j} \omega)\}$ dissipative for every $\Omega \in \Omega$ if and only if

1) There exists $\Omega_{0} \in \Omega$ such that $\Omega_{0} \star M$ is stable.

2) There exists a function $\Phi=\Phi^{*} \in \mathbf{R} \mathbf{L}_{\infty}$ such that $\Omega$ is $\left\{\Phi_{11}(\mathbf{j} \omega), \Phi_{12}(\mathbf{j} \omega), \Phi_{22}(\mathbf{j} \omega)\right\}$ dissipative for every $\Omega \in \boldsymbol{\Omega}$

3) There exists $\epsilon>0$ such that $\mathcal{L}_{\mathcal{P}}\left(M(\mathbf{j} \omega), \Phi_{11}(\mathbf{j} \omega), \ldots\right.$ $\left.\ldots, \Phi_{12}(\mathbf{j} \omega), \Phi_{22}(\mathbf{j} \omega), X(\mathbf{j} \omega), Y(\mathbf{j} \omega), Z(\mathbf{j} \omega), \epsilon\right) \geq 0$

Proof: The proof can be found in [22].

Theorem 3.1 presents necessary and sufficient conditions for the uncertain system $\Omega \star M$ to be $\{X(\mathbf{j} \omega), Y(\mathbf{j} \omega), Z(\mathbf{j} \omega)\}$ dissipative for every $\Omega \in \Omega$. This theorem requires

- the existence of $\Omega_{0} \in \Omega$ such that $\Omega_{0} \star M$ is stable;

- the verification of conditions 2) and 3) for every $\omega \in \overline{\mathbf{R}}^{+}$.

Satisfying condition 1) is not difficult and it is sufficient to take any $\Omega_{0} \in \Omega$ and test if $\Omega_{0} \star M$ is stable. If it is the case, we can continue testing conditions 2 ) and 3 ). Otherwise, we can conclude that $\Omega \star M$ is not stable for all $\Omega \in \Omega$.

Testing conditions 2) and 3) along the frequencies $\omega \in \overline{\mathbf{R}}^{+}$ is impossible from a practical point of view due to the infinity of tests to be performed.

To overcome this difficulty and as it is usual for LTI systems, a frequency by frequency approach can be performed without loss of generality [23] and a frequency griding $\mathcal{W}$ is defined where $\mathcal{W}$ is a finite set of frequencies.

In this case and given a frequency $\omega_{0} \in \mathcal{W}$, we define the static uncertain system $\Omega\left(\mathbf{j} \omega_{0}\right) \star M\left(\mathbf{j} \omega_{0}\right)$ where $M\left(\mathbf{j} \omega_{0}\right)$ is a given matrix and $\Omega\left(\mathbf{j} \omega_{0}\right) \in \boldsymbol{\Omega}\left(\mathbf{j} \omega_{0}\right)$ where $\boldsymbol{\Omega}\left(\mathbf{j} \omega_{0}\right)$ is given by

$$
\boldsymbol{\Omega}\left(\mathbf{j} \omega_{0}\right)=\left\{\Omega\left(\mathbf{j} \omega_{0}\right) \mid \Omega\left(\mathbf{j} \omega_{0}\right)=\underset{j}{\mathbf{b d i a g}}\left(\Omega_{j}\left(\mathbf{j} \omega_{0}\right)\right)\right\}
$$

Furthermore, the static uncertain system $\Omega\left(\mathbf{j} \omega_{0}\right) \star M\left(\mathbf{j} \omega_{0}\right)$ is said to be $\left\{X\left(\mathbf{j} \omega_{0}\right), Y\left(\mathbf{j} \omega_{0}\right), Z\left(\mathbf{j} \omega_{0}\right)\right\}$ dissipative if the condition (6) is respected with $\omega=\omega_{0}$ for every $\Omega\left(\mathbf{j} \omega_{0}\right) \in \mathbf{\Omega}\left(\mathbf{j} \omega_{0}\right)$ with $X\left(\mathbf{j} \omega_{0}\right)=X\left(\mathbf{j} \omega_{0}\right)^{*}$ and $Z\left(\mathbf{j} \omega_{0}\right)=Z\left(\mathbf{j} \omega_{0}\right)^{*}$.

Assuming the existence of $\Omega_{0} \in \Omega$ such that $\Omega_{0} \star M$ is stable and after introducing the frequency griding set $\mathcal{W}$, the robust performance analysis problem boils down to a set of matrix problems which can be interpreted as the robustness analysis of the static uncertain system $\Omega\left(\mathbf{j} \omega_{0}\right) \star M\left(\mathbf{j} \omega_{0}\right)$. Each of these problems is given by

Problem 3.1: Let $\Omega\left(\mathbf{j} \omega_{0}\right) \star M\left(\mathbf{j} \omega_{0}\right)$ be a static uncertain system where $M\left(\mathbf{j} \omega_{0}\right)$ is a given matrix and $\Omega\left(\mathbf{j} \omega_{0}\right) \in$ $\boldsymbol{\Omega}\left(\mathbf{j} \omega_{0}\right)$ where $\boldsymbol{\Omega}\left(\mathbf{j} \omega_{0}\right)$ is given by (7). Given the matrices $X\left(\mathbf{j} \omega_{0}\right), Y\left(\mathbf{j} \omega_{0}\right)$ and $Z\left(\mathbf{j} \omega_{0}\right)$ with $X\left(\mathbf{j} \omega_{0}\right)=X\left(\mathbf{j} \omega_{0}\right)^{*}$ and $Z\left(\mathbf{j} \omega_{0}\right)=Z\left(\mathbf{j} \omega_{0}\right)^{*}$. Test if the static uncertain system $\Omega\left(\mathbf{j} \omega_{0}\right) \star M\left(\mathbf{j} \omega_{0}\right)$ is $\left\{X\left(\mathbf{j} \omega_{0}\right), Y\left(\mathbf{j} \omega_{0}\right), Z\left(\mathbf{j} \omega_{0}\right)\right\}$ dissipative for every $\Omega\left(\mathbf{j} \omega_{0}\right) \in \boldsymbol{\Omega}\left(\mathbf{j} \omega_{0}\right)$. 
The following theorem gives necessary and sufficient conditions to perform the robust performance analysis of a static uncertain system $\Omega\left(\mathbf{j} \omega_{0}\right) \star M\left(\mathbf{j} \omega_{0}\right)$.

Theorem 3.2: Given the matrices $M\left(\mathbf{j} \omega_{0}\right), X\left(\mathbf{j} \omega_{0}\right), Y\left(\mathbf{j} \omega_{0}\right)$ and $Z\left(\mathbf{j} \omega_{0}\right)$ and the set $\boldsymbol{\Omega}\left(\mathbf{j} \omega_{0}\right)$ of Problem 3.1, the static uncertain system $\boldsymbol{\Omega}\left(\mathbf{j} \omega_{0}\right) \star M\left(\mathbf{j} \omega_{0}\right)$ is $\left\{X\left(\mathbf{j} \omega_{0}\right), Y\left(\mathbf{j} \omega_{0}\right), Z\left(\mathbf{j} \omega_{0}\right)\right\}$ dissipative for every $\Omega\left(\mathbf{j} \omega_{0}\right) \in \boldsymbol{\Omega}\left(\mathbf{j} \omega_{0}\right)$ if and only if

1) There exists a matrix $\Phi\left(\mathbf{j} \omega_{0}\right)=\Phi\left(\mathbf{j} \omega_{0}\right)^{*}$ such that $\Omega\left(\mathbf{j} \omega_{0}\right)$ is $\left\{\Phi_{11}\left(\mathbf{j} \omega_{0}\right), \Phi_{12}\left(\mathbf{j} \omega_{0}\right), \Phi_{22}\left(\mathbf{j} \omega_{0}\right)\right\}$ dissipative for every $\Omega\left(\mathbf{j} \omega_{0}\right) \in \boldsymbol{\Omega}\left(\mathbf{j} \omega_{0}\right)$

2) There exists $\epsilon>0$ such that $\mathcal{L}_{\mathcal{P}}\left(M\left(\mathbf{j} \omega_{0}\right), \Phi_{11}\left(\mathbf{j} \omega_{0}\right), \ldots\right.$ $\left.\ldots, \Phi_{12}\left(\mathbf{j} \omega_{0}\right), \Phi_{22}\left(\mathbf{j} \omega_{0}\right), X\left(\mathbf{j} \omega_{0}\right), Y\left(\mathbf{j} \omega_{0}\right), Z\left(\mathbf{j} \omega_{0}\right), \epsilon\right) \geq 0$

Proof: The proof is similar to the proof of Theorem 3.1 without considering the stability part.

In the sequel, for ease of notation, the frequency dependence will be dropped i.e. we write $\{X, Y, Z\}$ dissipativity instead of $\left\{X\left(\mathbf{j} \omega_{0}\right), Y\left(\mathbf{j} \omega_{0}\right), Z\left(\mathbf{j} \omega_{0}\right)\right\}$ dissipativity. Furthermore, if the static uncertain system $\Omega \star M$ is $\{X, Y, Z\}$ dissipative for every $\Omega \in \Omega$, then the static uncertain system set $\Omega \star M$ is $\{X, Y, Z\}$ dissipative. Moreover, for the sake of simplicity, the static uncertain system set will be referred to as the static uncertain system.

Theorem 3.2 presents necessary and sufficient conditions for the static uncertain system $\Omega \star M$ to be $\{X, Y, Z\}$ dissipative. Testing these conditions is a convex optimization problem. Nevertheless, it is infinite dimensional since condition 1) has to be tested for all $\Omega \in \Omega$ which is impossible from a computational point of view.

In order to obtain a finite dimensional convex optimization problem, let us introduce the set

$$
\Phi_{\Omega}=\left\{\Phi_{\Omega}=\left(\begin{array}{cc}
\underset{j}{\operatorname{bdiag}}\left(\left(\Phi_{11}\right)_{j}\right) & \underset{j}{\operatorname{bdiag}}\left(\left(\Phi_{12}\right)_{j}\right) \\
\mathbf{b d i a g}\left(\left(\Phi_{12}^{*}\right)_{j}\right) & \underset{j}{\operatorname{bdiag}}\left(\left(\Phi_{22}\right)_{j}\right)
\end{array}\right)\right\}
$$

such that condition 1) is satisfied for all $\Omega \in \Omega$. Let us introduce as well the sets associated to each $\Omega_{j} \in \boldsymbol{\Phi}_{\boldsymbol{\Omega}_{j}}$ as

$$
\boldsymbol{\Phi}_{\Omega_{j}}=\left\{\Phi_{\Omega_{j}}=\left(\begin{array}{ll}
\left(\Phi_{11}\right)_{j} & \left(\Phi_{12}\right)_{j} \\
\left(\Phi_{12}^{*}\right)_{j} & \left(\Phi_{22}\right)_{j}
\end{array}\right)\right\}
$$

Furthermore, for each $\Omega_{j}$, let us define $\mathcal{B}_{\Omega_{j}}^{\text {diss }}$ sets of conically independent elements $\Phi_{j}^{k} \in \boldsymbol{\Phi}_{\boldsymbol{\Omega}_{j}}$ with $k \in\left\{1, \cdots, n_{j}^{d}\right\}$, i.e. each $\Phi_{j}^{k}$ cannot be expressed as the conic combination of $\Phi_{j}^{l}, l \in\left\{1, \cdots, n_{j}^{d}\right\} \backslash\{k\}$. Please note, since $\Phi_{j}^{k} \in \boldsymbol{\Phi}_{\boldsymbol{\Omega}_{j}}$ for all $k \in\left\{1, \cdots, n_{j}^{d}\right\}$, the uncertainty $\Omega_{j}$ is $\left\{\left(\Phi_{11}\right)_{j}^{k},\left(\Phi_{12}\right)_{j}^{k},\left(\Phi_{22}\right)_{j}^{k}\right\}$ dissipative with

$$
\left(\begin{array}{ll}
\left(\Phi_{11}\right)_{j}^{k} & \left(\Phi_{12}\right)_{j}^{k} \\
\left(\Phi_{12}^{*}\right)_{j}^{k} & \left(\Phi_{22}\right)_{j}^{k}
\end{array}\right)=\Phi_{j}^{k} \in \mathcal{B}_{\Omega_{j}}^{\text {diss }}
$$

Then, let us define $\boldsymbol{\Phi}\left(\mathcal{B}_{\Omega}^{\text {diss }}\right)$ a set of block diagonal conic combinations of the elements of $\mathcal{B}_{\Omega_{j}}^{\text {diss }}$ as



Since $\boldsymbol{\Phi}_{\Omega}$ is a convex cone, we have $\boldsymbol{\Phi}\left(\mathcal{B}_{\Omega}^{\text {diss }}\right) \subseteq \boldsymbol{\Phi}_{\Omega}$.

A counterpart of Theorem 3.2 with sufficient conditions only is given in the following corollary.

Corollary 3.1: Given the matrices $M, X, Y$ and $Z$ and the set $\Omega$ of Problem 3.1 and if there exist basis sets $\mathcal{B}_{\Omega_{j}}^{\text {diss }}$ of conically independent elements $\Phi_{j}^{i}$ as in (8) for which $\Omega_{j}$ is $\left\{\left(\Phi_{11}\right)_{j}^{k},\left(\Phi_{12}\right)_{j}^{k},\left(\Phi_{22}\right)_{j}^{k}\right\}$ dissipative $\forall k$, then the static uncertain system $\Omega \star M$ is $\{X, Y, Z\}$ dissipative if there exists a $\Phi^{\Omega} \in \boldsymbol{\Phi}\left(\mathcal{B}_{\Omega}^{\text {diss }}\right)$ as in (9) and there exists $\epsilon>0$ such that

$$
\mathcal{L}_{\mathcal{P}}\left(M, \Phi_{11}^{\Omega}, \Phi_{12}^{\Omega}, \Phi_{22}^{\Omega}, X, Y, Z, \epsilon\right) \geq 0
$$

Proof: This is an immediate consequence of Theorem 3.2 after the parametrization of $\Phi$ as in (9).

Remark 3.2: In contrast with Theorem 3.2 and if there exist the basis $\mathcal{B}_{\Omega_{j}}^{\text {diss }}$, Corollary 3.1 presents sufficient conditions for the static uncertain system $\Omega \star M$ to be $\{X, Y, Z\}$ dissipative. Testing these conditions is a finite dimensional convex optimization problem under LMI constraints with $\alpha_{i j}$ as decision variables. It can be solved efficiently [2]. The consequence of introducing this parametrization $\boldsymbol{\Phi}\left(\mathcal{B}_{\Omega}^{\text {diss }}\right)$ is a possible conservatism. However, it can be reduced by an appropriate choice of $\mathcal{B}_{\Omega_{j}}^{\text {diss }}$, depending on the class of the uncertainty sets $\boldsymbol{\Omega}_{j}$. The conservatism depends also on how precise each element of $\mathcal{B}_{\Omega_{j}}^{\text {diss }}$ characterizes $\boldsymbol{\Omega}_{j}$. Furthermore, the larger $\mathcal{B}_{\Omega_{j}}^{\text {diss }}$ sets are, the less conservative results can be obtained.

In the case where the uncertainty set $\Omega$ is an elementary uncertainty set i.e. $\boldsymbol{\Omega}=\boldsymbol{\Delta}$, the associated basis can be easily obtained from [3] and [4] and it will be denoted by $\mathcal{B}_{\Delta}^{\text {diss }}$. In this case $\mathcal{B}_{\Delta}^{\text {diss }}$ can be defined as the well-known D or DG scaling sets according to (9).

\section{Application to uncertain LSS}

A solution to Problem 2.1 can be obtained by applying the tools presented in this section if the LSS is expressed as (5). After gathering all the different $\Delta_{j}^{i}$ in $\widetilde{\Delta}$, defining the global uncertainty set $\widetilde{\boldsymbol{\Delta}}$, while the different $M_{j}^{i}$ and the interconnections are gathered in $\widetilde{M}$ using LFT algebra, the global system $T_{1}^{1}$ will be given by

with

$$
T_{1}^{1}=\widetilde{\Delta} \star \widetilde{M}
$$

$$
\widetilde{\Delta}=\underset{i}{\operatorname{bdiag}}\left(\underset{j}{\operatorname{bdiag}}\left(\Delta_{j}^{i}\right)\right), \quad \Delta_{j}^{i} \in \boldsymbol{\Delta},
$$


Please note that $\widetilde{M} \in \mathbf{R} \mathbf{H}_{\infty}$. It is a direct consequence of the fact that the LSS is designed to be stable (in the nominal case).

As explained earlier, if there exists $\widetilde{\Delta}_{0} \in \widetilde{\boldsymbol{\Delta}}$ such that $\widetilde{\Delta}_{0} \star \widetilde{M}$ is stable and after considering a finite frequency set $\mathcal{W}$, then Problem 2.1 can be treated as a set of robust performance analysis problems of the static uncertain system $\widetilde{\Delta} \star \widetilde{M}$. In these problems, we denote $\gamma_{\omega_{0}}$ as an upper bound on the system maximum singular value $\bar{\sigma}(\bullet)$ over all the uncertainties for a given frequency $\omega_{0} \in \mathcal{W}$. After dropping the frequency dependence, each of these problems is given by

Problem 3.2: Let the static uncertain system $T_{1}^{1}=\widetilde{\Delta} \star \widetilde{M}$ where $\widetilde{M}$ is a given matrix and $\widetilde{\Delta} \in \widetilde{\Delta}$ a set of the uncertain matrices $\widetilde{\Delta}$. Given a frequency $\omega_{0}$, solve

$$
\begin{array}{cl}
\min _{\gamma_{\omega_{0}}} & \gamma_{\omega_{0}} \\
\text { subject to } & \bar{\sigma}\left(T_{1}^{1}\right)<\gamma_{\omega_{0}} \quad \forall \widetilde{\Delta} \in \widetilde{\boldsymbol{\Delta}}
\end{array}
$$

We can try to solve Problem 3.2 using a direct application of Corollary 3.1. Since $\widetilde{\Delta}$ is the block diagonal composition of elementary uncertainties $\Delta_{j}^{i} \in \boldsymbol{\Delta}$, the associated basis $\mathcal{B}_{\widetilde{\Delta}}^{\text {diss }}$ can easily be chosen from [3] and [4]

This approach will be referred to as the Direct LSS Robust Performance Analysis (Direct LSS RPA) method and it is summarized in the following corollary.

Corollary 3.2 (Direct LSS RPA): Given a frequency $\omega_{0}$, Problem 3.2 can be solved with the following optimization problem

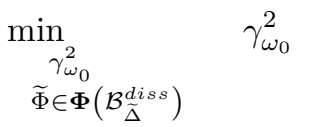

subject to

$$
\exists \epsilon>0 \text { s.t } \mathcal{L}_{\mathcal{P}}\left(\widetilde{M}, \widetilde{\Phi}_{11}, \widetilde{\Phi}_{12}, \widetilde{\Phi}_{22},-I, 0, \gamma_{\omega_{0}}^{2} I, \epsilon\right) \geq 0 .
$$

Proof: Corollary 3.2 is a direct application of Corollary 3.1 with $X=-I, Y=0$ and $Z=\gamma_{\omega_{0}}^{2} I$ (see Remark 3.1) and the minimization of $\gamma_{\omega_{0}}^{2}$ to obtain the lowest upper bound on $\bar{\sigma}\left(T_{1}^{1}\right)$ at a given frequency $\omega_{0}$.

Remark 3.3: If there are no performance signals ( $w_{1}^{1}$ and $z_{1}^{1}$ ), it is possible to investigate the robust stability of a network using this direct approach. The robust stability condition is given by $\left|\operatorname{det}\left(I-\widetilde{\Delta}(s) \widetilde{M}_{11}(s)\right)\right|>0$ for every $\widetilde{\Delta} \in \widetilde{\boldsymbol{\Delta}}$ and for every $s \in \overline{\mathbb{C}}^{+}$, see [24]. To test this condition, we have to find $\widetilde{\Delta}_{0} \in \widetilde{\boldsymbol{\Delta}}$ such that $\widetilde{\Delta}_{0}-\widetilde{M}_{11}$ is stable. Thereafter, we have to solve a set of matrix problems concerning the static interconnection $\widetilde{\Delta}\left(\mathbf{j} \omega_{0}\right)-\widetilde{M}_{11}\left(\mathbf{j} \omega_{0}\right)$ at each frequency $\omega_{0} \in \mathcal{W}$. Each of these problems can be solved using Corollary 3.2 by replacing condition (13) with $\mathcal{L}_{\mathcal{S}}\left(\widetilde{M}_{11}, \widetilde{\Phi}_{11}, \widetilde{\Phi}_{12}, \widetilde{\Phi}_{22}\right) \geq 0$. This approach will be referred to as the Direct LSS Robust Stability Analysis (LSS RSA).

As it will be shown later with numerical examples, the direct methods cannot be practically applied when the size of the LSS becomes too important. This is due to the important number of decision variables in the optimization problem in Corollary 3.2 which leads to a dramatic increase of the computation time.
In the next section, we propose a method that allows to take advantage of the hierarchical structure to practically investigate the robust performance of uncertain LSS within a reasonable computation time.

\section{HIERARCHICAL APPROACH}

In the previous section, we have shown that the performance can be characterized using dissipativity properties. Nevertheless, Corollary 3.1 reveals that to ensure robust performance, defined by the $\{X, Y, Z\}$ dissipativity property, it is sufficient (and sometimes necessary) to exhibit a dissipativity property, defined by $\Phi^{\Omega} \in \mathbf{\Phi}\left(\mathcal{B}_{\Omega}^{\text {diss }}\right)$, satisfied by all $\Omega \in \boldsymbol{\Omega}$ i.e. $\Omega$ is $\left\{\Phi_{11}^{\Omega}, \Phi_{12}^{\Omega}, \Phi_{22}^{\Omega}\right\}$ dissipative such that

$$
\mathcal{L}_{\mathcal{P}}\left(M, \Phi_{11}^{\Omega}, \Phi_{12}^{\Omega}, \Phi_{22}^{\Omega}, X, Y, Z, \epsilon\right) \geq 0
$$

holds true. Therefore, the dissipativity properties are suitable to characterize both uncertainty and performance.

Corollary 3.1 allows to characterize the performance with $\{X, Y, Z\}$ dissipativity property knowing that all the uncertainties $\Omega$ are $\left\{\Phi_{11}^{\Omega}, \Phi_{12}^{\Omega}, \Phi_{22}^{\Omega}\right\}$ dissipative where $\Phi^{\Omega}$ is constructed from the basis of the uncertainty $\Omega_{j}$ as in (8) and (9). Thereafter, if we have a method, which will use necessarily Corollary 3.1, that allows to find a basis $\mathcal{B}_{T}^{\text {diss }}$ for the static system $T=\Omega \star M$ from the basis $\mathcal{B}_{\Omega}^{\text {diss }}$ of the uncertainty, then Problem 3.2, which corresponds to Problem 2.1 at a given frequency $\omega_{0}$ (as explained in the previous section), can be solved efficiently in a hierarchical manner. This method will be known as "basis propagation" and it consists in propagating the basis from one hierarchical level to another starting from level $l$ where the uncertainty basis is known since $\Omega_{j}^{i}=\Delta_{j}^{i}$. This basis propagation method will be performed from basis of level $i$ to basis of level $i-1$ until reaching level 1 where the objective is to minimize $\gamma_{\omega_{0}}$.

The proposed Hierarchical Robust Performance Analysis (HRPA) approach is summarized in the following algorithm

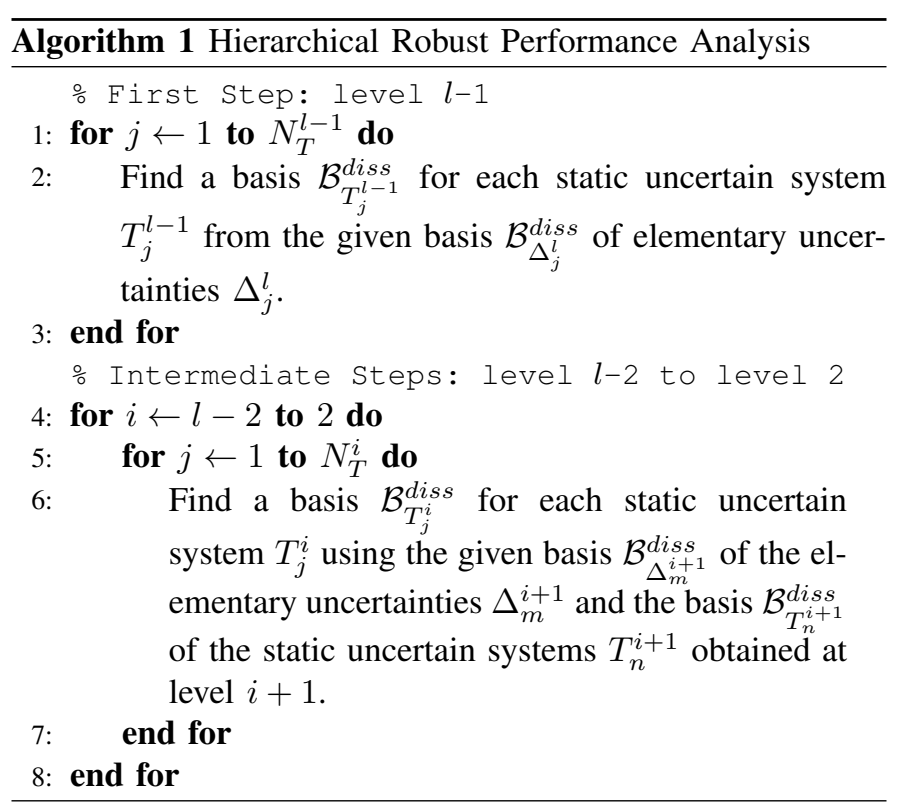




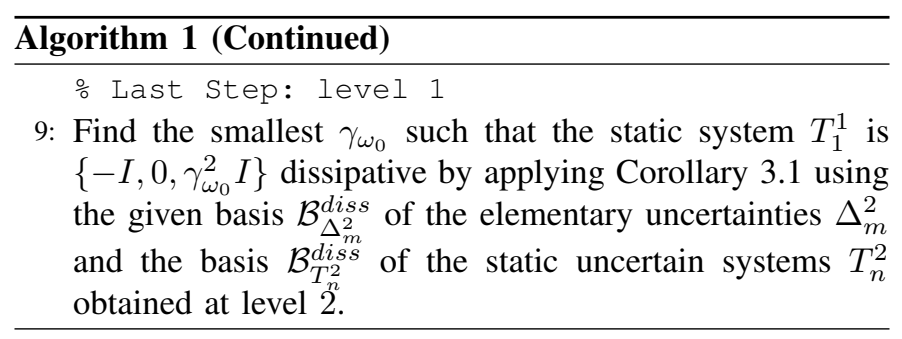

Remark 4.1: The robust stability problem of Remark 3.3 can be solved efficiently using basis propagation. As explained in Remark 3.3, the robust stability problem boils down to a set of matrix problems at each frequency $\omega_{0} \in \mathcal{W}$. Each of these problems can be solved using Algorithm 1 by replacing condition (10) of Corollary 3.1 in the last step of Algorithm 1 with $\mathcal{L}_{\mathcal{S}}\left(\left(M_{11}\right)_{1}^{1}, \Phi_{11}^{\Omega}, \Phi_{12}^{\Omega}, \Phi_{22}^{\Omega}\right) \geq 0$. This will define the Hierarchical Robust Stability Analysis Algorithm. Therefore, Algorithm 1 can be used for both certification: stability and performance after considering the corresponding static system robustness analysis problems.

Remark 4.2: Algorithm 1 allows to investigate the performance and the stability of uncertain LSS in an efficient manner after considering the corresponding static systems problems. The global LMI given in Corollary 3.2 will be replaced with several hierarchical LMI of Corollary 3.1 linked with appropriate condition in the next hierarchical levels. Furthermore, given a hierarchical level $i$, the different uncertain systems $T_{\bullet}^{i}$ do not interfere with each other since their interconnections appear in level $i-1$ and levels below. Therefore, the analysis performed at each level $i$ (the performance analysis of the different $T_{\bullet}^{i}$ ) can be performed separately and in parallel. The consequence of this parallel analysis will be a very important decrease of the computation time as it will be shown in subsection VI-B.

Remark 4.3: In the case of parametric uncertainties and when the size of the uncertain LSS is not too important such that Corollary 3.2 can be practically applied, the basis $\mathcal{B}_{\tilde{\Delta}}^{\text {diss }}$ can be chosen in the form of the well-known DG scaling from [3]. In order to reduce the conservatism resulting from choosing this parametrization, the basis $\mathcal{B}_{\widetilde{\Delta}}^{\text {diss }}$ can be chosen in the from of DGL scaling from [25] instead of DG scaling. In this case and since the basis is larger, the number of decision variables is more important and Corollary 3.2 may not be practically applied even if the size of the LSS is not too important. Nevertheless, Algorithm 1 allows to overcome this issue. Since the analysis in each level is performed on small size systems $T_{j}^{i}$, it is possible to choose the basis $\mathcal{B}_{\Delta_{m}^{i+1}}^{\text {diss }}$ in the from of DGL scaling. The consequence will be less conservative results in the overall analysis compared to those when using the hierarchical approach with DG scaling.

In the next section, we present three types of elements for the basis $\mathcal{B}_{T:}^{\text {diss }}$ and we formulate the problems of computing each one of them as a convex optimization problem allowing to minimize its size measure.

\section{Practical Formulation and Computation of BASIS ELEMENTS}

In the previous section, we revealed how it is possible to solve Problem 2.1 (subsequently Problem 3.2) with Algorithm 1 using basis propagation from level $i$ to level $i-1$ with the assumption that we are able to find the basis elements of each static uncertain system given the basis of its uncertainty. However, the conservatism of the overall result highly depends on the choice of the propagated basis. For this reason, it is important to define and compute the "best" basis for a given uncertain system. In the sequel, we consider a static uncertain system $T$ such that $z=T w$ as in (5) with a given uncertainty basis $\mathcal{B}_{\Omega}^{\text {diss }}$.

Corollary 3.1 gives sufficient conditions to obtain $\{X, Y, Z\}$ dissipativity property for the static uncertain system $T$ by solving the following feasibility optimization problem

$$
\mathcal{L}_{\mathcal{P}}\left(M, \Phi_{11}^{\Omega}, \Phi_{12}^{\Omega}, \Phi_{22}^{\Omega}, X^{k}, Y^{k}, Z^{k}, \epsilon\right) \geq 0
$$

with $\Phi^{\Omega} \in \boldsymbol{\Phi}\left(\mathcal{B}_{\Omega}^{\text {diss }}\right)$ is of the form (9) with a priori known $\mathcal{B}_{\Omega_{j}}^{\text {diss }}$. This feasibility optimization problem is convex and can be solved efficiently. The obtained $\{X, Y, Z\}$ dissipativity property will define an element of the basis $\mathcal{B}_{T}^{\text {diss }}$. However, in order to ensure that this characterization is the best, it is important to define a size measure for each element i.e. characterize $T$ with the optimal $\{X, Y, Z\}$ in the sense that this size is minimized. In addition, one can characterize the static uncertain system $T$ not with just one dissipativity property but with $N$ different dissipativity properties which will be used to construct the basis $\mathcal{B}_{T}^{\text {diss }}$

$$
\left(\begin{array}{cc}
X^{k} & Y^{k} \\
\left(Y^{k}\right)^{*} & Z^{k}
\end{array}\right) \in \mathcal{B}_{T}^{\text {diss }}, \forall k=1, \ldots, N^{d}
$$

In order to construct the "best" basis $\mathcal{B}_{T}^{\text {diss }}$, all its elements should be conically independent and capture information, of different nature, characterizing the static uncertain system $T$ such as gain or phase information.

For the sake of simplicity, the term "static" will be dropped and the static uncertain system will be referred to as the uncertain system.

In this section, for every frequency $\omega_{0}$ and in order to have a geometric interpretation of each element of $\mathcal{B}_{T}^{\text {diss }}$, we characterize the uncertain system $T$ in the signals space using the system input and output signals $w$ and $z$. Within this context, a system $T$ is said to be $\{X, Y, Z\}$ dissipative if

$$
\left(\begin{array}{c}
z \\
w
\end{array}\right)^{*}\left(\begin{array}{cc}
X & Y \\
Y^{*} & Z
\end{array}\right)\left(\begin{array}{c}
z \\
w
\end{array}\right)>0
$$

Therefore, the search of each element of $\mathcal{B}_{T}^{\text {diss }}$ consists in the following: find $\{X, Y, Z\}$ dissipativity property such that (14) is ensured for all non null input signals $w$ with its resulting output $z=\Omega \star M w$ for all $\Omega \in \boldsymbol{\Omega}$.

\section{A. Ellipsoid}

If $X<0$, constraint (14) rewrites in the signals space as

$$
\left(z-z_{c}\right)^{*}(-X)\left(z-z_{c}\right)<w^{*}\left(Z-Y^{*} X^{-1} Y\right) w
$$


with $z_{c}=-X^{-1} Y w$ and $\left(Z-Y^{*} X^{-1} Y\right)$ is hermitian positive definite matrix since $X<0$ and constraint (14) holds.

Then, for all non null input signal $w$, the corresponding output signal $z$ belongs to the ellipsoid $\mathcal{E}_{w}\left(n_{z}\right.$ dimensional space) centered at $z_{c}$ and characterized with $\mathcal{P}_{w}$ such that

$$
\mathscr{E}_{\mathcal{P}_{w}}=\left\{z \in \mathbb{C}^{n_{z}} \mid\left(z-z_{c}\right)^{*} \mathcal{P}_{w}\left(z-z_{c}\right)<1\right\}
$$

where the matrix $\mathcal{P}_{w}$ determines how far the ellipsoid extends in every direction. $\mathcal{P}_{w}$ is given by

$$
\mathcal{P}_{w}=\frac{-X}{w^{*}\left(Z-Y^{*} X^{-1} Y\right) w}
$$

We are now interested in finding $X, Y$ and $Z$ corresponding to the smallest ellipsoid $\mathscr{E}_{\mathcal{P}_{w}}$ for all inputs such that ${ }^{1}\|w\|=1$ and the uncertain system $\Omega \star M$ is $\{X, Y, Z\}$ dissipative. For this purpose, a size measure $v$ for the ellipsoid $\mathscr{E}_{\mathcal{P}_{w}}$ can be defined as its volume $v=\operatorname{vol}\left(\mathscr{E}_{\mathcal{P}_{w}}\right)$, which is given by

$$
v=\beta \sqrt{\operatorname{det}\left(\mathcal{P}_{w}^{-1}\right)}
$$

where $\beta$ is a positive scalar which depends on $n_{z}$.

Problem 5.1: Let $\Omega \star M$ be an uncertain system. Find $X, Y$ and $Z$ which

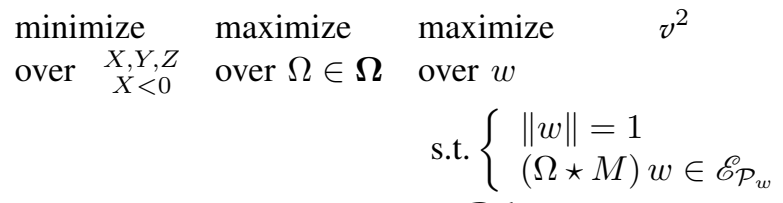

Theorem 5.1: An upper bound $\widetilde{v_{o p t}}$ on $v_{\text {opt }}$ optimal value of Problem 5.1 can be obtained by finding $X, Y, Z, \Phi_{11}^{\Omega}$, $\Phi_{12}^{\Omega}$ and $\Phi_{22}^{\Omega}$ with $\Phi^{\Omega}=\left(\begin{array}{cc}\Phi_{11}^{\Omega} & \Phi_{12}^{\Omega} \\ \left(\Phi_{12}^{\Omega}\right)^{*} & \Phi_{22}^{\Omega}\end{array}\right) \in \boldsymbol{\Phi}\left(\mathcal{B}_{\Omega}^{\text {diss }}\right)$ that minimize

$$
\log \left(\operatorname{det}\left(-X^{-1}\right)\right)
$$

subject to

1) $\exists \epsilon>0 \quad$ s.t $\quad \mathcal{L}_{\mathcal{P}}\left(M, \Phi_{11}^{\Omega}, \Phi_{12}^{\Omega}, \Phi_{22}^{\Omega}, X, Y, Z, \epsilon\right) \geq 0$

2) $\left(\begin{array}{ll}I & 0 \\ 0 & 0\end{array}\right) \geq\left(\begin{array}{cc}Z & Y^{*} \\ Y & X\end{array}\right)$.

The upper bound $\widetilde{v_{o p t}}$ is given by $\widetilde{v_{o p t}}=\beta \sqrt{\operatorname{det}\left(-\widetilde{X}^{-1}\right)}$ where $\widetilde{X}=\operatorname{argmin} \log \left(\operatorname{det}\left(-X^{-1}\right)\right)$ such that condition 1) and condition 2 ) of Theorem 5.1 hold.

This optimization problem is a determinant maximization under linear matrix inequality constraints [26] and is convex.

\section{Proof: Problem 5.1 rewrites

$\begin{array}{ll}\underset{\operatorname{minimize}}{\operatorname{maximize}} \quad \begin{array}{l}\text { maximize } \\ \text { over } X, Y, Z\end{array} & \begin{array}{l}v^{2} \\ \text { over } \Omega \in \Omega\end{array} \quad \text { over }\|w\|=1 \\ \text { subject to } & \boldsymbol{\Omega} \star M \text { is }\{X, Y, Z\} \text { dissipative }\end{array}$

Let us introduce the logarithm function on $v^{2}$, which is strictly increasing according to its argument

$\log \left(v^{2}\right)=2 \log (\beta)+\log \left(\operatorname{det}\left(-X^{-1}\right)\right)+\log \left(w^{*}\left(Z-Y^{*} X^{-1} Y\right) w\right)$

\footnotetext{
${ }^{1}$ The normalization of the input signal $w$ is absorbed in $\mathcal{P}_{w}$.
}

Maximizing $\log \left(v^{2}\right)$ over the inputs $\|w\|=1$ and with $\bar{\sigma}$ as the maximal singular value of $\left(Z-Y^{*} X^{-1} Y\right)$, it is possible to write

$$
\max _{\|w\|=1} \log \left(v^{2}\right)=2 \log (\beta)+\log \left(\operatorname{det}\left(-X^{-1}\right)\right)+\log (\bar{\sigma})
$$

Since $\left(Z-Y^{*} X^{-1} Y\right)$ is hermitian definite positive, then its maximal singular value is equal to its largest eigenvalue $\lambda_{\max }$. We thus have $\lambda_{\max } I \geq\left(Z-Y^{*} X^{-1} Y\right)$. Furthermore, as a dissipativity property is defined up to a strictly positive multiplicative coefficient and as $\{X, Y, Z\}$ dissipativity defines the same ellipsoid as $\{\tau X, \tau Y, \tau Z\}$ dissipativity for any $\tau>0$. Then, one can search for $X, Y$ and $Z$ such that $\lambda_{\max }=1$ without loss of generality. Since $\beta$ is a constant, the optimization problem is equivalent to

$$
\begin{array}{ll}
\underset{\text { minimize }}{\operatorname{maximize}} \quad \operatorname{mag}\left(\operatorname{det}\left(-X^{-1}\right)\right) \\
\text { over } X, Y, Z & \text { over } \Omega \in \Omega \\
\text { subject to } & \Omega \star M \text { is }\{X, Y, Z\} \text { dissipative } \\
& I \geq\left(Z-Y^{*} X^{-1} Y\right)
\end{array}
$$

The last optimization problem is solved by applying Corollary 3.1 which gives condition 1) of Theorem 5.1 while condition 2) is obtained by applying Schur's lemma [27] on $I \geq\left(Z-Y^{*} X^{-1} Y\right)$. Please note that since Corollary 3.1 presents sufficient conditions, we are only able to compute an upper bound $\widetilde{v_{\text {opt }}}$ on the optimal volume $v_{\text {opt }}$.

Remark 5.1: The interest of finding a bounded and connected set as an ellipsoid, for which belongs the output $z$, is to characterize the gain of the uncertain system $T$. At each frequency $\omega_{0}$, the frequency response of the uncertain system is embedded in this ellipsoid i.e. it is possible to compute boundaries for the system gain at this frequency $\omega_{0}$.

\section{B. Band}

In the signal space and if $X=0$, constraint (14) rewrites as $\xi^{*} z-\eta>0$ with $\xi=2 Y w\|2 Y w\|^{-1}$ and $\eta=-w^{*} Z w\|2 Y w\|^{-1}$. This last inequality expresses that for a given non null input signal $w$, the output signal $z$ belongs to a half plane which is characterized by the hyperplane: $\left\{z \mid \xi^{*} z=\eta\right\}$ where $\xi$ is a vector normal to the hyperplane and $\eta$ is twice the distance of the hyperplane to the origin (the dot product of any point of the hyperplane with $\xi$ ). In addition to a half plane, it is possible to define a band $\mathscr{B}_{\left(\eta_{1}, \eta_{2}\right)_{w}}$ as the intersection of two parallel half planes with the same normal direction $\xi$ but opposite sign, i.e. two parallel half planes defined by two dissipativity properties $\left\{0, Y, Z_{1}\right\}$ and $\left\{0,-Y, Z_{2}\right\}$, that is

$$
\mathscr{B}_{\left(\eta_{1}, \eta_{2}\right)_{w}}=\left\{\begin{array}{l|l}
z \in \mathbb{C}^{n_{z}} & \begin{array}{l}
\xi^{*} z-\eta_{1}>0 \\
-\xi^{*} z-\eta_{2}>0
\end{array}
\end{array}\right\}
$$

with $\xi=\frac{2 Y w}{\|2 Y w\|}, \eta_{1}=-\frac{w^{*} Z_{1} w}{\|2 Y w\|}$ and $\eta_{2}=-\frac{w^{*} Z_{2} w}{\|2 Y w\|}$.

Our objective now is to find the band $\mathscr{B}_{\left(\eta_{1}, \eta_{2}\right)_{w}}$ with the smallest size measure, for all inputs such that $\|w\|=1$ and provided that the uncertain system $\Omega \star M$ is $\left\{0, Y, Z_{1}\right\}$ 
and $\left\{0,-Y, Z_{2}\right\}$ dissipative. We can define a weighted size measure $d$ as the weighted width of the band

$$
d=\|2 Y w\|\left|\eta_{2}-\eta_{1}\right|
$$

Problem 5.2: Let $\Omega \star M$ be an uncertain system. Find $Y$, $Z_{1}$ and $Z_{2}$ which

minimize maximize maximize $d$

over $Y, Z_{1}, Z_{2} \quad$ over $\Omega \in \boldsymbol{\Omega}$ over $w$

$$
\text { subject to }\left\{\begin{array}{l}
\|w\|=1 \\
(\Omega \star M) w \in \mathscr{B}_{\left(\eta_{1}, \eta_{2}\right)_{w}}
\end{array}\right.
$$

Theorem 5.2: An upper bound $\widetilde{d_{o p t}}$ on $d_{o p t}$ the optimal value of Problem 5.2 can be obtained by finding $Y, Z_{1}, Z_{2}$, $\left(\Phi_{11}^{\Omega}\right)^{i},\left(\Phi_{12}^{\Omega}\right)^{i}$ and $\left(\Phi_{22}^{\Omega}\right)^{i}$ with $\left(\Phi^{\Omega}\right)^{i}=\left(\begin{array}{cc}\left(\Phi_{11}^{\Omega}\right)^{i} & \left(\Phi_{12}^{\Omega}\right)^{i} \\ \left(\Phi_{12}^{\Omega}\right)^{i^{*}} & \left(\Phi_{22}^{\Omega}\right)^{i}\end{array}\right) \in$ $\mathbf{\Phi}\left(\mathcal{B}_{\Omega}^{\text {diss }}\right)$ where $i=\{1,2\}$ that minimize

$$
d^{2}
$$

\section{subject to}

$$
\begin{aligned}
& \text { 1) } \exists \epsilon_{1}>0 \text { s.t. } \mathcal{L}_{\mathcal{P}}\left(M,\left(\Phi_{11}^{\Omega}\right)^{1},\left(\Phi_{12}^{\Omega}\right)^{1},\left(\Phi_{22}^{\Omega}\right)^{1}, 0, Y, Z_{1}, \epsilon_{1}\right) \geq 0 ; \\
& \text { 2) } \exists \epsilon_{2}>0 \text { s.t. } \mathcal{L}_{\mathcal{P}}\left(M,\left(\Phi_{11}^{\Omega}\right)^{2},\left(\Phi_{12}^{\Omega}\right)^{2},\left(\Phi_{22}^{\Omega}\right)^{2}, 0,-Y, Z_{2}, \epsilon_{2}\right) \geq 0 ; \\
& \text { 3) }\left(\begin{array}{cc}
d^{2} I & \left(Z_{1}-Z_{2}\right)^{*} \\
\left(Z_{1}-Z_{2}\right) & I
\end{array}\right) \geq 0 .
\end{aligned}
$$

The upper bound $\widetilde{d_{o p t}}$ is given by $\widetilde{d_{o p t}}=\sqrt{\operatorname{argmin} d^{2}}$ such that conditions 1 and 2 of Theorem 5.2 hold.

This optimization problem is the minimization of a linear cost under LMI constraints [27].

\section{Proof: Problem 5.2 rewrites}

$$
\begin{aligned}
& \text { minimize maximize maximize }\left|w^{*}\left(Z_{1}-Z_{2}\right) w\right| \\
& \text { over } Z_{1}, Z_{2} \quad \text { over } \Omega \in \Omega \quad \text { over }\|w\|=1 \\
& \begin{array}{ll}
\text { subject to } & \Omega \star M \text { is }\left\{0, Y, Z_{1}\right\} \text { dissipative } \\
& \Omega \star M \text { is }\left\{0,-Y, Z_{2}\right\} \text { dissipative }
\end{array}
\end{aligned}
$$

The maximum of $\left|w^{*}\left(Z_{1}-Z_{2}\right) w\right|$ over $\|w\|=1$ is equal to d constrained by

$$
\left(Z_{1}-Z_{2}\right)^{*}\left(Z_{1}-Z_{2}\right) \leq d^{2} I .
$$

The optimization is thus equivalent to

$\begin{array}{lc}\begin{array}{l}\text { minimize } \\ \text { over } Z_{1}, Z_{2}\end{array} & \begin{array}{r}\text { maximize } \\ \text { over } \Omega \in \Omega\end{array} \\ & \Omega \star M \text { is }\left\{0, Y, Z_{1}\right\} \text { dissipative } \\ \text { subject to } & \Omega \star M \text { is }\left\{0,-Y, Z_{2}\right\} \text { dissipative } \\ & \left(Z_{1}-Z_{2}\right)^{*}\left(Z_{1}-Z_{2}\right) \leq d^{2} I\end{array}$

Finally, condition 1) and condition 2) of Theorem 5.2 are obtained by applying Corollary 3.1 while condition 3 ) is obtained by applyin Schur's lemma [27] on $d^{2} I-$ $\left(Z_{1}-Z_{2}\right)^{*}\left(Z_{1}-Z_{2}\right) \geq 0$. Again, since Corollary 3.1 presents sufficient conditions, we are only able to compute an upper bound $\widetilde{d_{o p t}}$ on the optimal width $d_{o p t}$.

\section{Cone Sector}

The phase uncertainty presents another important characterization of the uncertain system behavior. In contrast with the system gain, and beside for single input single output systems, there is no unique definition of multiple input multiple output systems phase. Furthermore, taking into account uncertainties in the system makes the phase characterization more complicated. It is possible to compare the direction variation between the input and the output signals to measure the phase of a system by measuring input direction variation introduced by the system. Furthermore, within this context, the phase uncertainty is characterized using the notion of numerical range as shown in [20] and [28]. The numerical range of a complex matrix $\Gamma$ is defined to be a compact and convex set of $\mathbb{C}$ and it is given by [29]

$$
\mathcal{N}(\Gamma)=\left\{w^{*} z \mid z=\Gamma w, w \in \mathbb{C}^{n_{w}} \text { and }\|w\|=1\right\}
$$

In order to define the phase of an uncertain system $\Omega \star M$, the numerical range is extended to the union of numerical ranges $\mathcal{N}(\Omega \star M)$ for any $\Omega \in \Omega$. Let us define in the complex plane the cone sector centered at the origin and containing all these numerical ranges. It is defined by a spread angle $\alpha$ such that $0<\alpha<\pi$ and the angle $\beta$ measured between the bisectrix of $\alpha$ and the real axis direction. Please refer to [20] for more details. The angle $\beta$ can be set to zero by introducing a scaling matrix $\Psi \in \mathbb{C}^{n_{z} \times n_{w}}$. More generally, it is possible to introduce an offset $z_{c}$ characterized with a matrix $C \in \mathbb{C}^{n_{z} \times n_{w}}$ such that $z_{c}=C w$ and search for the cone sector $\mathscr{C}_{(0, \alpha)}$ containing $\mathcal{N}\left(\Psi^{*}(\Omega \star M-C)\right)$ with

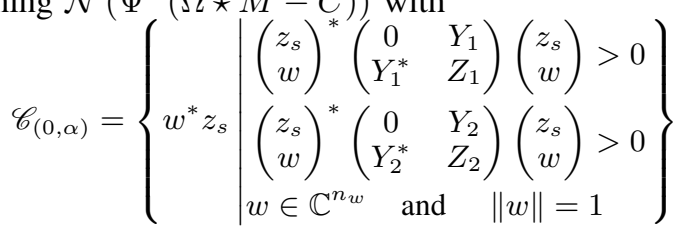

where

- $z_{s}$ belongs to the signal set for which the dot product with the signal $w$ gives the cone sector $\mathscr{C}_{(0, \alpha)}$;

- $Y_{1}=\Psi\left(I+\mathbf{j} \cot \left(\frac{\alpha}{2}\right) I\right)$ and $Z_{1}=-\left(Y_{1}^{*} C+C^{*} Y_{1}\right)$;

- $Z_{2}=\Psi\left(I-\mathbf{j} \cot \left(\frac{\alpha}{2}\right) I\right)$ and $Z_{2}=-\left(Y_{2}^{*} C+C^{*} Y_{2}\right)$. with $0<\alpha<\pi$. We are now interested in finding $Y_{i}$ and $Z_{i}$ corresponding to the smallest $\mathscr{C}_{(0, \alpha)}$ for all inputs such that $\|w\|=1$. This inclusion ensures that the uncertain system $\Omega \star M$ is $\left\{0, Y_{1}, Z_{1}\right\}$ and $\left\{0, Y_{2}, Z_{2}\right\}$ dissipative. This problem can be solved by finding $\Psi$ such that the union of the numerical ranges $\mathcal{N}\left(\Psi^{*}(\Omega \star M-C)\right)$ is in the right half plane and then search for $\alpha$. Furthermore, to obtain the smallest cone, a size measure $a$ for $\mathscr{C}_{(0, \alpha)}$ is needed. It can be defined as the tangent of the angle $\alpha$

$$
a=\tan \left(\frac{\alpha}{2}\right)
$$

Problem 5.3: Let $\Omega \star M$ be an uncertain system and given a complex matrix $C$. Find $\Psi$ and $\alpha$ such that the union of the numerical ranges $\mathcal{N}\left(\Psi^{*}(\Omega \star M-C)\right)$ is in the right half plane and which

$$
\begin{aligned}
& \text { minimize maximize maximize } a \\
& \text { over } \Psi, a \quad \text { over } \Omega \in \Omega \quad \text { over } w \\
& \text { s.t. }\left\{\begin{array}{l}
\|w\|=1 \\
\mathcal{N}\left(\Psi^{*}(\Omega \star M-C)\right) \subset \mathscr{C}_{(0, \alpha)}
\end{array}\right.
\end{aligned}
$$


Theorem 5.3: The union of numerical ranges $\mathcal{N}\left(\Psi^{*}(\Omega \star M-C)\right)$ of Problem 5.3 is located in the right half plane if $\exists \Psi$ and $\exists \widehat{\Phi} \in \boldsymbol{\Phi}\left(\mathcal{B}_{\Omega}^{\text {diss }}\right)$ and $\exists \epsilon>0$ such that

$$
\left(\begin{array}{c}
M \\
I
\end{array}\right)^{*} B\left(\begin{array}{c}
M \\
I
\end{array}\right) \geq 0
$$

where

$$
B=\left(\begin{array}{cccc}
-\widehat{\Phi}_{22}^{\Omega} & 0 & -\left(\widehat{\Phi}_{12}^{\Omega}\right)^{*} & 0 \\
0 & -\epsilon I & 0 & \Psi \\
-\widehat{\Phi}_{12}^{\Omega} & 0 & -\widehat{\Phi}_{11}^{\Omega} & 0 \\
0 & \Psi^{*} & 0 & -\left(\Psi^{*} C+C^{*} \Psi\right)-\epsilon I
\end{array}\right)
$$

Furthermore, an upper bound $\widetilde{a_{o p t}}$ on $a_{o p t}$, optimal value of Problem 5.3 , can be obtained by finding $a, \widehat{\Phi}^{\Omega}$ and $\left(\widetilde{\Phi}^{\Omega}\right)^{i}$ with $\widehat{\Phi}^{\Omega}+a^{-1}\left(\widetilde{\Phi}^{\Omega}\right)^{1} \in \Phi\left(\mathcal{B}_{\Omega}^{\text {diss }}\right)$ and $\widehat{\Phi}^{\Omega}-a^{-1}\left(\widetilde{\Phi}^{\Omega}\right)^{2} \in$ $\boldsymbol{\Phi}\left(\mathcal{B}_{\Omega}^{\text {diss }}\right)$ that minimize

$a$

subject to

1) $a\left(\begin{array}{c}M \\ I\end{array}\right)^{*} B\left(\begin{array}{c}M \\ I\end{array}\right)+\left(\begin{array}{c}M \\ I\end{array}\right)^{*} A_{1}\left(\begin{array}{c}M \\ I\end{array}\right) \geq 0$
2) $a\left(\begin{array}{c}M \\ I\end{array}\right)^{*} B\left(\begin{array}{c}M \\ I\end{array}\right)+\left(\begin{array}{c}M \\ I\end{array}\right)^{*} A_{2}\left(\begin{array}{c}M \\ I\end{array}\right) \geq 0$

3) Condition (18)

where $A_{i}=(-1)^{i-1} \times \ldots$

$$
\cdots \times\left(\begin{array}{cccc}
-\left(\widetilde{\Phi}_{22}^{\Omega}\right)^{i} & 0 & -\left(\left(\widetilde{\Phi}_{12}^{\Omega}\right)^{i}\right)^{*} & 0 \\
0 & 0 & 0 & \mathbf{j} \Psi \\
-\left(\widetilde{\Phi}_{12}^{\Omega}\right)^{i} & 0 & -\left(\widetilde{\Phi}_{11}^{\Omega}\right)^{i} & 0 \\
0 & (\mathbf{j} \Psi)^{*} & 0 & \mathbf{j}\left(\Psi^{*} C-C^{*} \Psi\right)
\end{array}\right) .
$$

The upper bound $\widetilde{a_{o p t}}$ is given by $\widetilde{a_{o p t}}=\operatorname{argmin} a$ such that conditions 1), 2) and 3) of Theorem 5.3 hold.

Minimizing $a$ in Theorem 5.3 such that conditions 1), 2) and 3) hold is a generalized eigenvalues problem which has been proved that it is a quasiconvex optimization problem [27] and it can be solved efficiently [30].

Proof: The union of the numerical ranges $\mathcal{N}\left(\Psi^{*}(\Omega \star M-C)\right)$ is in the right half plane if and only if

$$
\operatorname{Re}\left(\mathcal{N}\left(\Psi^{*}(\Omega \star M-C)\right)\right) \geq 0 .
$$

By (16), which defines the numerical range, the previous condition rewrites as

$$
\left(\begin{array}{c}
z \\
w
\end{array}\right)^{*}\left(\begin{array}{cc}
0 & \Psi \\
\Psi^{*} & -\left(\Psi^{*} C+C^{*} \Psi\right)
\end{array}\right)\left(\begin{array}{c}
z \\
w
\end{array}\right) \geq 0
$$

where $z=\Omega \star M$. This constraint expresses that the uncertain system $\Omega \star M$ is $\left\{0, \Psi,-\left(\Psi^{*} C+C^{*} \Psi\right)\right\}$ dissipative for all $\Omega \in \Omega$. By applying Corollary 3.1, (19) is implied by (18) with $\widehat{\Phi} \in \boldsymbol{\Phi}\left(\mathcal{B}_{\Omega}^{\text {diss }}\right)$. Then, the minimization of $a$ is well-posed when the union of numerical ranges $\mathcal{N}\left(\Psi^{*}(\Omega \star M-C)\right)$ is in the right half plane, that is $0<\frac{\alpha}{2}<\frac{\pi}{2}$. With $a=\tan \left(\frac{\alpha}{2}\right), Y_{i}$ and $Z_{i}$ introduced in (17) become

$$
\begin{aligned}
& Y_{i}=\Psi+(-1)^{i-1} \mathbf{j} a^{-1} \Psi \\
& Z_{i}=-\left(\Psi^{*} C+C^{*} \Psi\right)+(-1)^{i} \mathbf{j} a^{-1}\left(\Psi^{*} C-C^{*} \Psi\right)
\end{aligned}
$$

The problem of minimizing $a$ rewrites

$$
\begin{array}{lll}
\operatorname{minimize} & \operatorname{maximize} & \operatorname{maximize} \\
\text { over } \Psi, \boldsymbol{a} & \text { over } \Omega \in \Omega & \text { over }\|w\|=1
\end{array}
$$

subject to $\Omega \star M$ is $\left\{0, Y_{1}, Z_{1}\right\}$ dissipative

where $Y_{i}$ and $Z_{i}$ are given in (20). Nevertheless, since the conditions of Corollary 3.1 are only sufficient, we are able to compute only an upper bound $\widetilde{a}$ on $a_{o p t}$, solution of Problem 5.3. Applying Corollary 3.1 gives

$$
\begin{aligned}
& \mathcal{L}_{\mathcal{P}}\left(M,\left(\Phi_{11}^{\Omega}\right)^{1},\left(\Phi_{12}^{\Omega}\right)^{1},\left(\Phi_{22}^{\Omega}\right)^{1}, 0, Y_{1}, Z_{1}, \epsilon\right) \geq 0 \\
& \mathcal{L}_{\mathcal{P}}\left(M,\left(\Phi_{11}^{\Omega}\right)^{2},\left(\Phi_{12}^{\Omega}\right)^{2},\left(\Phi_{22}^{\Omega}\right)^{2}, 0, Y_{2}, Z_{2}, \epsilon\right) \geq 0
\end{aligned}
$$

with $\left(\Phi^{\Omega}\right)^{1} \in \boldsymbol{\Phi}\left(\mathcal{B}_{\Omega}^{\text {diss }}\right),\left(\Phi^{\Omega}\right)^{2} \in \boldsymbol{\Phi}\left(\mathcal{B}_{\Omega}^{\text {diss }}\right) . Y_{i}$ and $Z_{i}$ are given in (20). However, since $a^{-1}$ is multiplied by $\Psi$, the optimization problem is bilinear in $a^{-1}$ and $\Psi$. In general, optimization problems involving bilinear matrix inequalities are NP hard, except if e.g. it belongs to the particular sub-class of generalized eigenvalue problems which are quasiconvex optimization problems and they can be solved efficiently [27]. In the sequel, we reveal that the minimization of $a$ can be rewritten as a generalized eigenvalue problem. To this purpose, it is necessary to transform the inequalities (21) in order to obtain bilinear terms which are $a$ multiplied by positive semidefinite matrices. To this purpose, we enforce $\left(\Phi^{\Omega}\right)^{i}=\widehat{\Phi}^{\Omega}$ with $i \in\{1,2\}$. Nevertheless, fixing this structure to $\left(\Phi^{\Omega}\right)^{i}$ will increase the conservatism. The latter can be reduced with a more appropriate choice of $\left(\Phi^{\Omega}\right)^{i}$ such as $\left(\Phi^{\Omega}\right)^{1}=$ $\widehat{\Phi}^{\Omega}+a^{-1}\left(\widetilde{\Phi}^{\Omega}\right)^{1}$ and $\left(\Phi^{\Omega}\right)^{2}=\widehat{\Phi}^{\Omega}-a^{-1}\left(\widetilde{\Phi}^{\Omega}\right)^{2}$. This choice is interesting in the sense that $\widehat{\Phi}^{\Omega}$ will be used to make $\mathcal{N}\left(\Psi^{*}(\Omega \star M-C)\right)$ in the right half plane while $\left(\widetilde{\Phi}^{\Omega}\right)^{i}$ will ensure extra degree of freedom to minimize $\widetilde{a}$. Developing (21) and factorizing $a^{-1}$ give

$$
\begin{aligned}
& \left(\begin{array}{c}
M \\
I
\end{array}\right)^{*} B\left(\begin{array}{c}
M \\
I
\end{array}\right)+a^{-1}\left(\begin{array}{c}
M \\
I
\end{array}\right) A_{1}\left(\begin{array}{c}
M \\
I
\end{array}\right) \geq 0 ; \\
& \left(\begin{array}{c}
M \\
I
\end{array}\right)^{*} B\left(\begin{array}{c}
M \\
I
\end{array}\right)+a^{-1}\left(\begin{array}{c}
M \\
I
\end{array}\right) A_{2}\left(\begin{array}{c}
M \\
I
\end{array}\right) \geq 0 .
\end{aligned}
$$

Since $0<\alpha<\pi$, then $a=\tan \left(\frac{\alpha}{2}\right)>0$ and we obtain

$$
\begin{aligned}
& a\left(\begin{array}{c}
M \\
I
\end{array}\right)^{*} B\left(\begin{array}{c}
M \\
I
\end{array}\right)+\left(\begin{array}{c}
M \\
I
\end{array}\right) A_{1}\left(\begin{array}{c}
M \\
I
\end{array}\right) \geq 0 ; \\
& a\left(\begin{array}{c}
M \\
I
\end{array}\right)^{*} B\left(\begin{array}{c}
M \\
I
\end{array}\right)+\left(\begin{array}{c}
M \\
I
\end{array}\right)^{*} A_{2}\left(\begin{array}{c}
M \\
I
\end{array}\right) \geq 0 .
\end{aligned}
$$

Minimizing $a$ with the latter conditions is a generalized eigenvalue problem since condition (18) holds. Please note that since Corollary 3.1 presents sufficient conditions, we are only able to compute an upper bound $\widetilde{a_{o p t}}$ on the optimal $a_{o p t}$. 
Remark 5.2: The interest of finding a cone sector is to be able to characterize the phase variation by embedding the numerical range of the uncertain system inside the cone sector. This phase uncertainty information can be very important and critical in some applications such as the active control of vibrations. Please note that for SISO system, the offset $z_{c}=C w$, with $\|w\|=1$, boils down to one point $z_{c}=C$ and it is possible to interpret the cone sector $\mathscr{C}_{(0, \alpha)}$ containing $\mathcal{N}\left(\Psi^{*}(\Omega \star M-C)\right)$ as the cone sector $\mathscr{C}_{\left(z_{c}, \alpha, \arg (\Psi)\right)}$ containing $\mathcal{N}(\Omega \star M)$. Furthermore, if $C=0$ and $\boldsymbol{\Omega}=0$, the numerical range boils down into one point and the cone sector is nothing else than the phase of the nominal SISO system, according to the origin, at a given frequency $\omega_{0}$.

In the next section we present two applications examples to investigate the robustness of uncertain LSS using the hierarchical approach.

\section{APPLICATIONS OF THE HIERARCHICAL APPROACH}

\section{A. Performance analysis of PLL network}

We consider in this first example the performance analysis of the active clock distribution network of [31]. It is composed of $N=16$ mutually synchronized Phase Locked Loop (PLL) delivering clock signals to the chip. In order to synchronize all the network, the PLLs exchange information through an interconnection structure. This example is suitable for illustration of the proposed hierarchical analysis approach as the performance is naturally evaluated in the frequency domain.

1) PLL network description: The description of the $N$ PLLs frequency responses are given by

$$
T_{j}\left(\mathbf{j} \omega_{0}\right)=\frac{k_{j}\left(\mathbf{j} \omega_{0}+a_{j}\right)}{-\omega^{2}+k_{j} \mathbf{j} \omega_{0}+k_{j} a_{j}} \quad \forall j \in\{1, \ldots, N\}
$$

where $k_{j}$ and $a_{j}$ are the PLL parameters and $\omega_{0}$ is the current frequency defined by griding. Due to the manufacturing process, technological dispersions are inevitable and the PLL parameters $k_{j}$ and $a_{j}$ are uncertain: $k_{j} \in[0.76,6.84] \times 10^{4}$ and $a_{j} \in[91.1,273.3]$. Furthermore, all the PLLs are homogeneous i.e. have the same description and uncertainty set $\Delta$. Therefore, and after normalization, it is possible to present the PLLs as the interconnection of a certain part and uncertain one

$$
T_{j}\left(\mathbf{j} \omega_{0}\right)=\Delta_{j} \star M_{P L L} \quad \Delta_{j} \in \boldsymbol{\Delta} \quad \forall j \in\{1 \ldots, N\}
$$

where $\boldsymbol{\Delta}$ is given by

$$
\boldsymbol{\Delta}=\left\{\Delta=\left(\begin{array}{cc}
\delta_{k} & 0 \\
0 & \delta_{a}
\end{array}\right) \quad \delta_{k} \in \mathbb{R}, \quad \delta_{a} \in \mathbb{R} \quad\|\Delta\|_{\infty}<1\right\}
$$

The information exchange between PLLs is modeled by an interconnection matrix $M_{\text {int }}$ defined in the equation (13) of [18].

Therefore, the PLL network has a three level hierarchical structure ans it is a sub-class of the LSS of (3) and (4). The

\begin{tabular}{|c|c|c|}
\hline$l$ & \multicolumn{2}{|l|}{3} \\
\hline$N_{T}^{i}$ & \multicolumn{2}{|c|}{$N_{T}^{1}=1, N_{T}^{2}=N$} \\
\hline $\mathcal{N}_{\Delta}\left(M_{j}^{i}\right)$ & $\begin{array}{l}\mathcal{N}_{\Delta}\left(M_{j}^{2}\right) \\
\mathcal{N}_{\Delta}\left(M_{1}^{1}\right) \\
\end{array}$ & $\begin{array}{l}=\{j\} \quad \forall j \in\left\{1, \ldots, N_{T}^{2}\right\} \\
=\emptyset\end{array}$ \\
\hline $\mathcal{N}_{T}\left(M_{j}^{i}\right)$ & $\begin{array}{l}\mathcal{N}_{T}\left(M_{\bullet}^{2}\right) \\
\mathcal{N}_{T}\left(M_{1}^{1}\right) \\
\end{array}$ & $\begin{array}{l}=\emptyset \\
=\{1, \ldots, N\}\end{array}$ \\
\hline$M_{j}^{i}$ & \multicolumn{2}{|c|}{$M_{1}^{1}=M_{i n t}, M_{j}^{2}=M_{P L L} \forall j \in\left\{1, \ldots, N_{T}^{2}\right\}$} \\
\hline
\end{tabular}
different parameters of this hierachical structure are presented in TABLE I.
TABLE I: Three level hierarchical structure parameters

The network performance is characterized by its global input and output signals $w_{g}$ and $z_{g}$ using the global frequency response magnitude bound (more details in [18] and [19]).

2) Hierarchical Approach: Algorithm 1 is applied in two steps.

- Local step: compute a basis $\mathcal{B}_{T_{j}}^{\text {diss }}$ for each PLL by applying Corollary 3.1 using the given basis $\mathcal{B}_{\Delta_{j}}^{\text {diss }}$ of the elementary uncertainties $\Delta_{j}$. In order to construct the basis $\mathcal{B}_{T_{j}}^{\text {diss }}$, we consider several elements

- ellipsoid: we can use Theorem 5.1 to find a disc ${ }^{2}$ center and an upper bound on the optimal radius;

- band: we can use Theorem 5.2 to find an upper bound on the band width with free orientation;

- cone sector: given a scalar $C$, we can use Theorem 5.3 to find an upper bound of the cone angle.

Please note that since all the PLLs are homogeneous, the basis $\mathcal{B}_{T_{j}}^{\text {diss }}$ obtained for one PLL is valid for all the 16 PLL. Therefore, computation time of the local step is equal to computation time required to find the basis elements for one PLL.

- Global step : compute the smallest $\gamma_{\omega_{0}}$ such that the network, denoted $T_{1}^{1}$, is $\left\{-I, 0, \gamma_{\omega_{0}}^{2} I\right\}$ dissipative by applying Corollary 3.1 using the basis $\mathcal{B}_{T_{j}}^{\text {diss }}$ obtained in local step. In this step, one can combine the elements of $\mathcal{B}_{T_{j}}^{\text {diss }}$ to characterize each PLL by: ellipsoid alone, ellipsoid and band, ellipsoid and cone, etc. Then, propagate these characterizations to investigate the performance of the network in the global step.

3) Results:

- Local step : In this step, we are interested in finding three elements of the basis $\mathcal{B}_{T_{j}}^{\text {diss }}$ : ellipsoid, band and cone sector. The frequency response of a PLL is presented in Fig. 2 in the complex plane for a given frequency $\omega_{0}=480 \mathrm{rad} / \mathrm{s}$. The red dot is the nominal frequency response and the green dots represent a sampling of the uncertain frequency responses obtained for a sampling of $a_{j}$ and $k_{j}$ for illustration purposes only. The three elements of $\mathcal{B}_{T_{j}}^{\text {diss }}$ are interpreted in geometric terms and presented in Fig. 2. The obtained disc is pre-

\footnotetext{
${ }^{2}$ In single input single output case, the ellipsoid boils down to a disc.
} 


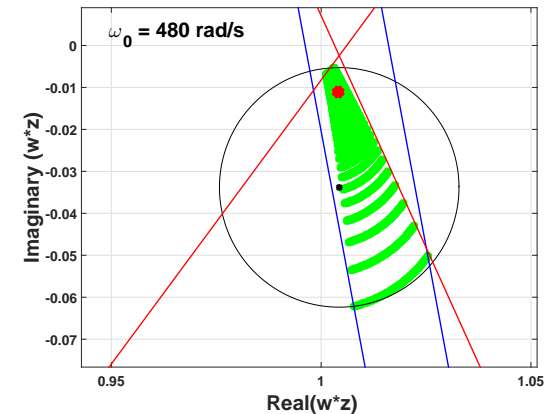

Fig. 2: Three elements of $\mathcal{B}_{T_{j}}^{\text {diss }}$ characterizing each PLL



Fig. 3: The PLL network performance analysis

sented in black with its center. We can see that the radius is minimized. The blue lines represent the obtained band with a free direction and the band width is also minimized. The red lines represent the obtained cone sector with its center $C$ fixed as $C=c_{d i s c}+\mathbf{j} \times 1.1 \times r_{d i s c}$ where $c_{d i s c}$ and $r_{d i s c}$ are the disc center and radius respectively and the cone angle is minimized. Please note that since the uncertainty $\Delta$ is parametric and in order to reduce the conservatism, the basis $\mathcal{B}_{\Delta}^{\text {diss }}$ is chosen in the form of DGL scaling from [25], see Remark 4.3.

- Global step: The performance analysis results of the PLL network are presented in Fig. 3 and summarized in TABLE II. Please note that the direct approach corresponds to the approach of Corollary 3.2.

4) Discussion: All the analysis reveal that the PLL network is able to track a ramp as the slope of frequency response magnitude at low frequencies is $40 \mathrm{~dB} / \mathrm{dec}$ (see [31] for more details) and the performance specifications are satisfied.

\begin{tabular}{|l|c|c|}
\hline Approach & Maximum peak & Computation time \\
\hline Direct & $6.01 \mathrm{~dB}$ & $361.7 \mathrm{~s}$ \\
\hline Hierarchical: ellipsoid & $13.44 \mathrm{~dB}$ & $16.9 \mathrm{~s}$ \\
\hline Hierarchical: ellipsoid+band & $12.97 \mathrm{~dB}$ & $53.5 \mathrm{~s}$ \\
\hline Hierarchical: ellipsoid+cone & $6.45 \mathrm{~dB}$ & $115.9 \mathrm{~s}$ \\
\hline
\end{tabular}

TABLE II: Comparison between the different approaches
The direct approach presents the less conservative results with a maximum peak of $6.01 d B$ compared to the different hierarchical approaches. However, computation time is significant: 361.7s. TABLE II illustrates the trade-off between conservatism and computation time that can be set by the user with the hierarchical approach. Please note that TABLE II gives the overall computation time for each approach i.e. computation time required for both steps. When using the disc alone, the results are conservative but they are obtained faster. The results are less conservative when using the disc with the band, but they are obtained in more time. However the maximum peak is also important. When using the disc with a cone sector, the results are much less conservative. Actually, they are close to the results of the direct approach: the difference in the maximal peak value with direct approach is $+0.39 \mathrm{~dB}$, that corresponds to $4.73 \%$ of ratio. In addition, the results are obtained in $32.04 \%$ of the time needed for direct approach.

\section{B. Stability Analysis of a Chain of Uncertain Systems}

In this second example, we consider the stability analysis of a chain of uncertain systems taken from [16]. Our objective is to compare the computation time required in our hierarchical method, the direct analysis approach and the method proposed in [16].

1) Uncertain system chain description: Consider a chain of $N$ uncertain system $T_{j}$. The uncertainties are assumed to be scalar reals: $\delta_{1}, \ldots, \delta_{N}$ i.e. each system has one parametric uncertainty. The inputs and the outputs of each system $T_{j}$ are denoted $w_{j}$ and $z_{j}$.

For $j=\{2, \ldots, N-1\}$, each system $T_{j}$ has two inputs and two outputs i.e. $w_{j}, z_{j} \in \mathbb{C}^{2}$ while $w_{j}, z_{j} \in \mathbb{C}$ for $j=\{1, N\}$; hence it is possible, after normalization, to represent each uncertain system as

$$
T_{j}=\Delta_{j} \star M_{j} \quad \Delta_{j} \in \Delta
$$

with $\Delta$ is given by

$$
\boldsymbol{\Delta}=\left\{\Delta=\delta, \quad \delta \in \mathbb{R} \quad\|\Delta\|_{\infty}<1\right\} .
$$

Since each uncertain system $T_{j}$ is only connected to $T_{j-1}$ and to $T_{j+1}$, see Fig.4, the interconnections in this chain are defined by: $w_{j}^{2}=z_{j+1}^{1}$ and $w_{j}^{1}=z_{j-1}^{2}$ for $j=\{2, \ldots, N-1\}$ and by $w_{1}=z_{2}^{1}$ and $w_{N}=z_{N-1}^{2}$ for $j=\{1, N\}$. The global system is given by

$$
T_{\text {global }}=\operatorname{bdiag}_{j}\left(T_{j}\right) \star M_{\text {global }}
$$

where $M_{\text {global }}$ is the global interconnection matrix. This matrix is sparse and it corresponds to a chordal graph, please refer to [16] for more details.

The systems are generated randomly as explained in [16] where the authors considered three conditions that different systems should satisfy: each system has to be nominally and robustly stable and the chain of $N$ systems has to be nominally stable.

A possible application corresponding to the uncertain system chain of Fig. 4 is when an electric current is flowing through a line with a length that is not negligible compared to the 


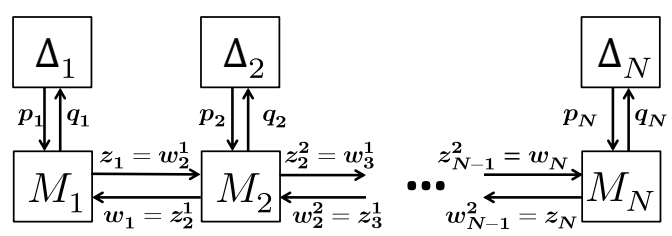

Fig. 4: Chain of $N$ uncertain systems

current wave length at the considered frequency. An example is a railway power supply line. Indeed, the line length can be more than $100 \mathrm{~km}$ while trains generate current harmonics at frequencies in the $\mathrm{kHz}$ range (wave length in tens of $\mathrm{km}$ ). These kind of systems are often modeled with cascaded blocks where each block models a short part of the line (this part should be of short length compared to the wave length). Usually blocks are modeled as quadripoles which are electrical circuits with two pairs of terminals to connect to external circuits, see [32] and the references within. Because of the modeling errors, the quadripole characteristic parameters are not precisely known and uncertainties should be considered to study the worst case stability scenario.

2) Hierarchical approach: Let us suppose, without loss of generality, that $N$ the number of systems in the chain is given by $N=2^{\nu}$ where $\nu$ is a positive integer. It is possible to consider a three levels hierarchical structure as in TABLE I, with $M_{1}^{1}=M_{\text {global }}$ and $M_{j}^{2}=M_{j}$ (the randomly defined), and perform the analysis in two steps as for the PLL application.

However, with this three level hierarchical structure, we will not exploit to the most the network structure which is sparse with a chordal pattern. To exploit this pattern, special optimization algorithms can be used as shown in [16]. Nevertheless, our hierarchical approach is flexible enough to capture this structure and exploit the chordal pattern. Since each system $T_{j}$ is only connected to its direct neighbors, let us define a two by two ${ }^{3}$ fully split hierarchical structure.

The chain of uncertain systems with two by two fully split hierarchical structure is a sub-class of the LSS of (3) and (4). The different parameters of this hierarchical structure are summarized in TABLE III.

Since the LSS of Fig. 4 has no input output performance signal, we will use the Hierarchical Robust Stability Analysis Algorithm of Remark 4.1. The new hierarchical analysis setup will be performed in several steps.

- First step : find a basis $\mathcal{B}_{T_{j}^{l-1}}^{\text {diss }}$ for each uncertain system $T_{j}^{l-1}$ by applying Corollary 3.1 using the given basis $\mathcal{B}_{\Delta_{j}^{l}}^{\text {diss }}$ of the elementary uncertainties $\Delta_{j}^{l}$.

- Intermediate steps : for every $i=l-2, \ldots, 2$ and for every $j=1, \ldots, N_{T}^{i}$, find a basis $\mathcal{B}_{T_{j}^{j}}^{\text {diss }}$ for each uncer-

\footnotetext{
${ }^{3}$ Except level $l$ and level $l-1$, we regroup systems two by two at each hierarchical level.
}

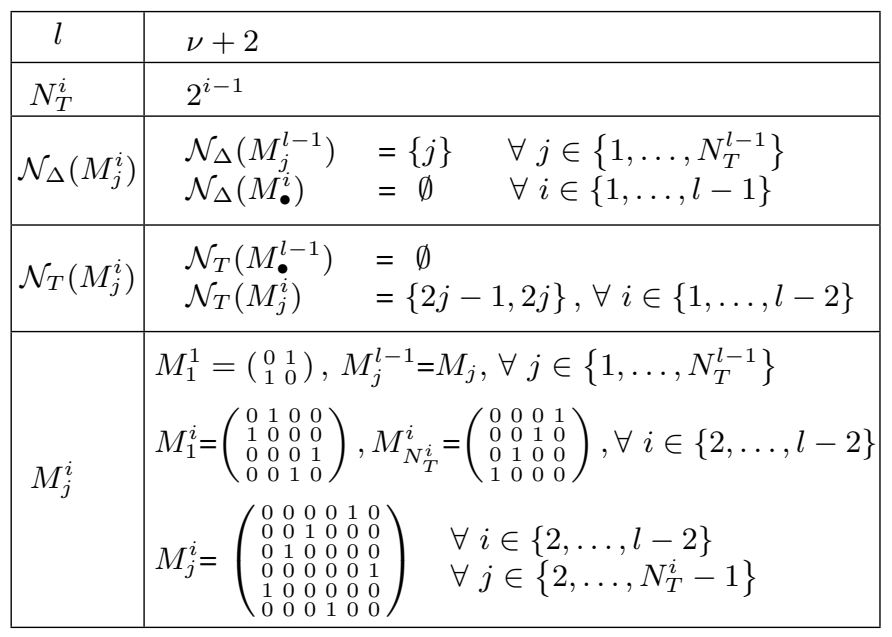

TABLE III: Two by two fully split hierarchical structure parameters

tain system $T_{j}^{i}$ using the basis $\mathcal{B}_{T_{n}^{i+1}}^{\text {diss }}$ of the uncertain systems $T_{n}^{i+1}$ obtained at level $i+1$.

- Last step : given the basis $\mathcal{B}_{T_{j}^{2}}^{\text {diss }}$ obtained at level 2, test if $\mathcal{L}_{\mathcal{S}}\left(M_{1}^{1}, \Phi_{11}^{\Omega}, \Phi_{12}^{\Omega}, \Phi_{22}^{\Omega},\right) \geq 0$.

The Robust Stability Analysis Algorithm (see Remark 4.1) will be used by considering only one element: ellipsoid at each level. Furthermore and as we have seen, the uncertain systems of each hierarchical level $i$ are interconnected at level $i-1$ and levels below, it is hence easy to perform the hierarchical approach using parallel computation as it is explained in Remark 4.2.

3) Results: The objective here is to compute the ratio between the time required in the direct and the hierarchical approach and to compare it to that obtained in [16]. Therefore, we will apply the different approaches: direct and parallel hierarchical only for a single frequency.

The analysis is performed for 10 realizations of the network presented earlier with different $N$. The evolution according to $N$ of the average CPU time required to perform the analysis of 10 realizations for each approach is plotted in Fig.5.

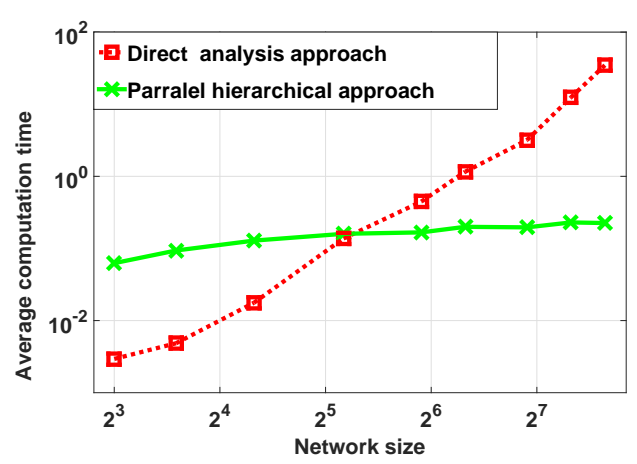

Fig. 5: Average CPU computation time versus $N$ 
Please note that in order to compare our results with those of [16], the basis $\mathcal{B}_{\widetilde{\Delta}}^{\text {diss }}$ is the D-scaling from [4].

The average CPU time required for the direct approach increases dramatically when increasing the size of the network and it can reach 37.40 s for a chain of 200 systems. For the hierarchical approach, the analysis is performed in a parallel manner at each hierarchical level. The required CPU time, at level $i$, is the maximum among all the CPU time required for the analysis of each subsystem $T_{j}^{i}$ and the global CPU time is the sum of the computation time of each level. The average CPU time required for $N=200$ is 0.2552 s which gives a ratio of 154 between the time required in direct and hierarchical approaches. For comparison, this ratio is 10 in [16].

Please note that for small size chains (less than approximately 36), the computation time of the hierarchical approach is more important compared to the direct approach. When the chain size is increasing, the computation time of the direct approach becomes more and more important compared to the hierarchical approach. This behavior can be explained by analyzing how the network size $N$ affects the computation time in both approach. The objective of the next section is to discuss how the computation time grows according to $N$.

\section{Computation time For a Sub-Class of UNCERTAIN LSS}

The hierarchical structure introduced in the second example of the previous section can be generalized by regrouping systems $n_{s}$ by $n_{s}$ instead of two by two, to obtain $n_{s}$ by $n_{s}$ fully split hierarchical structure. Without loss of generality, let us suppose that $N$ the number of systems in the chain is given by $N=n_{s}^{\kappa}$ where $\kappa$ is a positive integer.

The chain of uncertain systems with $n_{s}$ by $n_{s}$ fully split hierarchical structure is a sub-class of the LSS of (3) and (4). The different parameters of this hierarchical structure are given as in TABLE III with the following differences

- $l=\kappa+2$;

- $N_{T}^{i}=n_{s}^{i-1}$;

- $\mathcal{N}_{T}\left(M_{j}^{i}\right)=\left\{n_{s}(j-1)+1, \ldots, n_{s} j\right\}$;

- $M_{j}^{i}$, for $i \neq l-1$, are adapted according to $n_{s}$.

Furthermore, the stability problem considered in the previous section can be extended to a performance problem i.e. investigate if the chain is $\left\{X_{1}, Y_{1}, Z_{1}\right\}$ dissipative for all possible uncertainties by introducing the global performance signal $w_{g}$ and $z_{g}$ and adapting $M_{1}^{1}$.

For illustration purpose of the computation time, and without lost of generality, we suppose that all the uncertainties $\Delta_{j}^{l}$ are homogeneous. Furthermore, again and without lost of generality, we perform basis propagation in hierarchical approaches, from level $l-1$ to level 2 , with only one element of $\mathcal{B}_{T_{j}^{i}}^{\text {diss }}$ such as the ellipsoid.

The objective is to characterize the computation time required to perform robustness analysis, at a given frequency $\omega_{0}$, using the different approaches: direct, hierarchical and parallel hierarchical.

\section{Computation complexity}

Computation time of an algorithm characterizes how fast or slow this algorithm performs. This computation time depends on the algorithm complexity which is defined as a numerical function of the number of variables $n$.

Given an optimization problem under LMI constraints, the algorithmic complexity is $\mathcal{O}\left(n^{3}\right)$ when using the interior point method [30]. The computation time of an algorithm depends on implementation details such as: processor speed, instruction set, etc.. For this reason, in the sequel, the computation time is referred to as the algorithmic complexity.

\section{A. Direct approach}

For the direct analysis approach and given a dissipativity property $\left\{X_{1}, Y_{1}, Z_{1}\right\}$, the number of decision variables of the robust performance analysis is $\eta_{\left\{X_{1}, Y_{1}, Z_{1}\right\}}+\sum_{k=1}^{N} \eta_{\Delta_{k}}$ where $\eta_{\left\{X_{1}, Y_{1}, Z_{1}\right\}}$ is the number of decision variables corresponding to $\left\{X_{1}, Y_{1}, Z_{1}\right\}$ and $\eta_{\Delta_{k}}$ is the number of decision variables corresponding to each elementary uncertainty $\Delta_{k}$. In the case where all the uncertainties are homogeneous, all the $\eta_{\Delta_{k}}$ become $\eta_{\Delta}$ and the required computation time is given by

$$
T_{D A}=\mathcal{O}\left(\left(\eta_{\left\{X_{1}, Y_{1}, Z_{1}\right\}}+N \eta_{\Delta}\right)^{3}\right)
$$

which is cubic according to $N$.

\section{B. Hierarchical approach}

Given $l=\kappa+2$ and $N_{T}^{i}=n_{s}^{i-1}$. The total number of analysis to be performed for all levels is the sum of $\kappa+1$ first terms of a geometric progression, that is

$$
\underbrace{1}_{\text {level } 1}+\underbrace{n_{s}+n_{s}^{2}+\cdots+n_{s}^{\kappa-1}}_{\text {intermediate levels }}+\underbrace{n_{s}^{\kappa}}_{\text {level } l-1}=\frac{N n_{s}-1}{n_{s}-1}
$$

The algorithmic complexity of the hierarchical approach depends on the number of performed tests. The latter changes according to the considered hierarchical structure i.e. the number of levels and the number of analysis at each level

- each of the $N$ analysis problem, at level $l-1$, has $\left(\eta_{\Delta}+\eta_{\{X, Y, Z\}}\right)$ decision variables with $\eta_{\{X, Y, Z\}}$ is the number of decision variables corresponding to one $\{X, Y, Z\}$ dissipativity property to be propagated. The computation time is $N \mathcal{O}\left(\left(\eta_{\Delta}+\eta_{\{X, Y, Z\}}\right)^{3}\right)$.

- each of the $N \frac{1}{n_{s}-1}-\frac{n_{s}}{n_{s}-1}$ intermediate analysis problems, from level $l-2$ to level 2 , has $\left(n_{s}+\eta_{\{X, Y, Z\}}\right)$ decision variables ${ }^{4}$. The required computation time is $\left(N \frac{1}{n_{s}-1}-\frac{n_{s}}{n_{s}-1}\right) \mathcal{O}\left(\left(n_{s}+\eta_{\{X, Y, Z\}}\right)^{3}\right)$.

- at level 1 , the objective is to investigate if $T_{1}^{1}\left(\mathbf{j} \omega_{0}\right)$ is $\left\{X_{1}, Y_{1}, Z_{1}\right\}$ dissipative. The analysis problem

${ }^{4}$ Here $n_{s}$ corresponds to the number of $\alpha_{k j}$ of (9), with $k=1$ and $j \in\left\{1, \ldots, n_{s}\right\}$, corresponding to the $n_{s}$ dissipativity properties propagated from level $i+1$. 
has $\left(n_{s}+\eta_{\left\{X_{1}, Y_{1}, Z_{1}\right\}}\right)$ decision variables and the computation time is $\mathcal{O}\left(\left(n_{s}+\eta_{\left\{X_{1}, Y_{1}, Z_{1}\right\}}\right)^{3}\right)$.

Then the global computation time, at a given frequency $\omega_{0}$, of the hierarchical approach $T_{H A}$ is

$$
\begin{aligned}
T_{H A}= & N \mathcal{O}\left(\left(\eta_{\Delta}+\eta_{\{X, Y, Z\}}\right)^{3}\right)+\mathcal{O}\left(\left(n_{s}+\eta_{\left\{X_{1}, Y_{1}, Z_{1}\right\}}\right)^{3}\right) \\
& +\left(N \frac{1}{n_{s}-1}-\frac{n_{s}}{n_{s}-1}\right) \mathcal{O}\left(\left(n_{s}+\eta_{\{X, Y, Z\}}\right)^{3}\right)
\end{aligned}
$$

which is affine with respect to $N$.

\section{Parallel hierarchical approach}

In this approach, the computation time at each level $i$ is the maximum among computation time required for the analysis problems at this level $i$.

- at level $l-1$, the required computation time is $\mathcal{O}\left(\left(\eta_{\Delta}+\eta_{\{X, Y, Z\}}\right)^{3}\right)$

- from level $l-2$ to level 2 , since the number of intermediate levels is $l-3$ and since $(l-2)=\kappa$, that is $\log _{n_{s}}(N)$, the required computation time is $\left(\log _{n_{s}}(N)-1\right) \mathcal{O}\left(\left(n_{s}+\eta_{\{X, Y, Z\}}\right)^{3}\right)$

- at level 1 , the computation time is $\mathcal{O}\left(\left(n_{s}+\eta_{\left\{X_{1}, Y_{1}, Z_{1}\right\}}\right)^{3}\right)$

The global computation time required in this parallel hierarchical approach $T_{P H A}$ is

$T_{P H A}=\mathcal{O}\left(\left(\eta_{\Delta}+\eta_{\{X, Y, Z\}}\right)^{3}\right)+\mathcal{O}\left(\left(n_{s}+\eta_{\left\{X_{1}, Y_{1}, Z_{1}\right\}}\right)^{3}\right)$ $+\log _{n_{s}}(N) \mathcal{O}\left(\left(n_{s}+\eta_{\{X, Y, Z\}}\right)^{3}\right)-\mathcal{O}\left(\left(n_{s}+\eta_{\{X, Y, Z\}}\right)^{3}\right)$

which is logarithmic according to $N$.

We are now able to explain the results of Fig.5. Since the objective is to test the stability of the chain, $\eta_{\left\{X_{1}, Y_{1}, Z_{1}\right\}}=0$ and the computation time $T_{D A}$ becomes

$$
T_{D A}=\mathcal{O}\left(\left(N \eta_{\Delta}\right)^{3}\right)
$$

For the parallel hierarchical approach with $n_{s}=2$, the computation time $T_{P H A}$ becomes

$$
\begin{aligned}
T_{P H A} & =\mathcal{O}\left((2)^{3}\right) \log _{2}(N) \mathcal{O}\left(\left(2+\eta_{\{X, Y, Z\}}\right)^{3}\right) \\
& +\mathcal{O}\left(\left(\eta_{\Delta}+\eta_{\{X, Y, Z\}}\right)^{3}\right)
\end{aligned}
$$

The algorithmic complexity of the direct approach is cubic according to $N$. For $N<36$, the algorithmic complexity is not really costly from computation time point of view and the analysis can be performed relatively fast. In the parallel hierarchical approach, even if finding one $\{X, Y, Z\}$ for each uncertain systems is not costly (since we are dealing with small size systems), the overall algorithmic complexity is important compared to direct approach because of the multiplication term by $\log _{2}(N)$. However, when $N$ is increasing, the evolution of the logarithmic function is less important than the cubic evolution which makes $T_{D A}$ really significant compared to $T_{P H A}$. Fig.5 confirms this trend. Please note that the scales in this figure are logarithmic.
Remark 7.1: It is possible to perform the same analysis for the case when $N \neq n_{s}^{\kappa}$. The number of levels and systems at each level will change and the expressions of $T_{H A}$ and $T_{P H A}$ will be slightly different. However, the affine and logarithmic evolution of $T_{H A}$ and $T_{P H A}$ remain valid.

\section{CONCLUSION AND FUTURE WORK}

In this paper, robustness analysis of uncertain large scale systems with hierarchical structure is considered. In order to reduce the computational load, a hierarchical robust performance analysis algorithm is proposed. This algorithm performs several hierarchical analysis using basis propagation from one hierarchical level to another. We have also presented how to formulate and compute several basis elements. The efficiency of this algorithm is illustrated through two examples: PLL network and chain of uncertain systems. The proposed algorithm allows to establish a trade-off between conservatism and computation time. Furthermore, we discuss the computation time for a sub-class of uncertain LSS. In contrast with cubic evolution of computation time with respect to $N$ in the direct approach, the computation time grows in an affine manner in the hierarchical approach and logarithmic manner in the parallel hierarchical approach. Therefore, the hierarchical approaches are more suitable and adapted to perform the robustness analysis when $N$ becomes very significant. Nevertheless, in this paper, we did not discuss how it is possible to obtain hierarchical structures neither the advantage of one hierarchical structure with respect to another. Therefore, major future work directions are

- establish a systematic approach to obtain the optimal hierarchical structure with respect to computation time and conservatism;

- combine the hierarchical approach with other approaches as the one presented in [15] and [16] i.e. introduce specialized solvers into the hierarchical approach.

\section{REFERENCES}

[1] R. D. Braatz, P. M. Young, J. C. Doyle, and M. Morari, "Computational complexity of $\mu$ calculation," IEEE Trans. Aut. Control, vol. AC-39, no. 5, pp. 1000-1002, May 1994.

[2] S. Boyd and L. Vandenberghe, Convex Optimization. Cambridge University Press, 2004.

[3] M. K. H. Fan, A. L. Tits, and J. C. Doyle, "Robustness in the presence of mixed parametric uncertainty and unmodeled dynamics," IEEE Trans. Aut. Control, vol. 36, no. 1, pp. 25-38, 1991.

[4] J. Doyle, "Analysis of feedback systems with structured uncertainties," IEEE Proceddings, vol. 129-D, p. 242:250, 1982.

[5] A. Megretski and A. Rantzer, "System analysis via integral quadratic constraints," IEEE Trans. Aut. Control, vol. 42, no. 6, pp. 819-830, 1997.

[6] P. Moylan, "A connective stability result for interconnected passive systems," IEEE Trans. Aut. Control, pp. 812-813, August 1980.

[7] M. Vidyasagar, Input/Output Analysis of Large-Scale Interconnected Systems, ser. Lecture Notes in Control and Information Sciences. Berlin: Springer-Verlag, 1981, no. 29.

[8] I. Letas and G. Vinnicombe, "Scalabe decentralized robust stability certificates for networks of interconnected heterogenous dynamical systems," IEEE Trans. Aut. Control, vol. 51, no. 10, pp. 1613-1626, Oct 2006 
[9] I. Lestas and G. Vinnicombe, "Heterogeneity and scalability in group agreement protocols: Beyond small gain and passivity approaches," Automatica, vol. 46, pp. 1141-1151, 2010.

[10] J. A. Fax and R. M. Murray, "Information flow and cooperative control of vehicle formations," IEEE Trans. Aut. Control, vol. 49, no. 09, pp. 1465-1476, Sep 2004.

[11] R. Olfati-Saber, J. Fax, and R. Murray, "Consensus and cooperation in networked multi-agent systems," Proc. IEEE, vol. 95, no. 1, pp. 215 -233 , Jan. 2007.

[12] K.-K. K. Kim and R. D. Braatz., "On the robustness of interconnected or networked uncertain multi-agent systems," In 20th International Symposium on Mathematical Theory of Networks and Systems, 2012.

[13] C.-Y. Kao, U. Jönsson, and H. Fujioka, "Characterization of robust stability of a class of interconnected systems," Automatica, vol. 45, no. 1, pp. 217-224, 2009.

[14] U. Jönsson and C.-Y. Kao, "A scalable robust stability criterion for systems with heterogeneous LTI components," IEEE Trans. Aut. Control, vol. 55, no. 10, pp. 2219-2234, Oct 2010.

[15] M. S. Andersen, A. Hansson, S. H. Pakazad, and A. Rantzer, "Distributed robust stability analysis of interconnected uncertain systetems," Proc. IEEE Conf. on Decision and Control, pp. 1548-1553, Dec 2012.

[16] M. S. Andersen, S. H. Pakazad, A. Hansson, and A. Rantzer, "Robust stability analysis of sparsely interconnected uncertain systems," IEEE Trans. Aut. Control, vol. 59, no. 8, pp. 2151-2156, Aug 2014.

[17] M. G. Safonov, "Propagation of conic model uncertainty in hierarchical systems," IEEE Trans. Aut. Control, vol. 28, no. 6, pp. 701-709, Jun 1983.

[18] M. Dinh, A. Korniienko, and G. Scorletti, "Embedding of uncertainty propagation: Application to hierarchical performance analysis," in IFAC Joint Conference, 5th Symposium on System Structure and Control, Grenoble, France, February 2013, pp. 190-195.

[19] — - "Convex hierrachical analysis for the performance of uncertain large scale systems," Proc. IEEE Conf. on Decision and Control, pp. 5979- 5984, Dec 2014.

[20] K. Laib, A. Kornnienko, G. Scorletti, and F. Morel, "Phase IQC for the hierarchical performance analysis of uncertain large scale systems," Proc. IEEE Conf. on Decision and Control, pp. 5953-5958, Dec 2015.

[21] S. Skogestad and I. Postlethwaite, Multivariable Feedback Control, Analysis and Design. John Wiley \& Sons, Chichester, England, 1996.

[22] K. Laib, A. Kornnienko, M. Dinh, G. Scorletti, and F. Morel, "Hierarchical approach to investigate the robust performance of uncertain large scale systems," Tech. Rep. Laboratoire Ampère, Ecole Centrale de Lyon, Tech. Rep., 2016. [Online]. Available: https://hal.archives-ouvertes.fr/hal-01306918

[23] Y.-S. Chou, A. Tits, and V. Balakrishnan, "Stability multipliers and $\mu$ upper bounds: connections and implications for numerical verification of frequency domain conditions," IEEE Trans. Aut. Control, vol. 44, no. 5, pp. 906-913, 1999.

[24] S. Skogestad and I. Postlethwaite, Multivariable Feedback Control, Analysis and Design. John Wiley and Sons Chischester, 2005.

[25] G. Scorletti, X. Bombois, M. Barenthin, and V. Fromion, "Improved efficient analysis for systems with uncertain parameters," in Proc. IEEE Conf. on Decision and Control, New Orleans, dec. 2007, pp. 5038-5043.

[26] L. Vandenberghe, S. Boyd, and S.-P. Wu, "Determinant maximization with linear matrix inequality constraints," SIAM Journal on Matrix Analysis and Applications, vol. 19, pp. 499-533, 1996.

[27] S. Boyd, L. El Ghaoui, E. Feron, and V. Balakrishnan, Linear Matrix Inequalities in Systems and Control Theory, ser. Studies in Appllied Mathematics. Philadelphia, USA: SIAM, June 1994, vol. 15.

[28] A. Tits, V. Balakrishnan, and L. Lee, "Robustness under bounded uncertainty with phase information," IEEE Trans. Aut. Control, vol. 44, no. $1,1999$.

[29] D. H. Owens, "The numerical range: A tool for robust stability studies?" Syst. Control Letters, no. 3, pp. 153-158, Dec 1984.

[30] Y. Nesterov and A. Nemirovski, Interior Point Polynomial Methods in Convex Programming: Theory and Applications. SIAM, 1994, vol. 13.

[31] A. Korniienko, G. Scorletti, E. Colinet, E. Blanco, J. Juillard, and D. Galayko, "Control law synthesis for distributed multi-agent systems: Application to active clock distribution networks," in Proc. American Control Conf., San Francisco, June 2011, pp. 4691- 4696.
[32] C. Stackler, F. Morel, P. Ladoux, and P. Dworakowski, " 25 kV-50 Hz railway supply modelling for medium frequencies (0-5 kHz)," in 2016 International Conference on Electrical Systems for Aircraft, Railway, Ship Propulsion and Road Vehicles International Transportation Electrification Conference (ESARS-ITEC), Nov 2016, pp. 1-6.

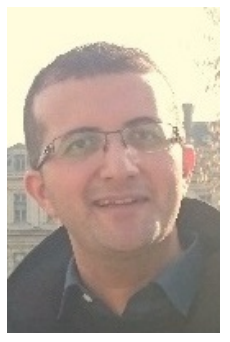

Khaled Laib received his Engineering degree from École Nationale Polytechnique, Algiers, Algeria, in 2011. He held a wireline field engineer position in Baker Hughes Inc for one year before joining Université Claude Bernard Lyon 1, France where he obtained the M.Sc. degree in control engineering, in 2013. He is currently a Ph.D student in the Ampère laboratory, École Centrale de Lyon, France. His research topics include robustness analysis, uncertain large-scale systems and uncertainty propagation, uncerain power flow analysis in power networks.

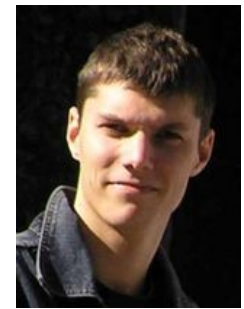

Anton Korniienko holding a $\mathrm{PhD}$ from the Université de Lyon in Automatic Control, Anton Korniienko is currently Assistant Professor in Automatic Control and Signal Processing at Ecole Centrale de Lyon. He obtained his degree from the National Technical University of Ukraine "Kiev Polytechnic Institute" and prepared his PhD in Control Theory applied to Microelectronic System Design at the French Atomic Energy and Alternative Energies Commission (CEA, LETI, Minatec) in Grenoble. His research topics are: design and control of large-scale network systems, multi-agents, decentralized control, robust control, uncertainty impacts.

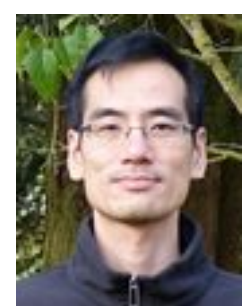

Marc Dinh was born in 1979. He received his engineering degree from École Nationale Supérieure d'Ingénieurs de Caen, and Ph.D. in Automatic Control from Universitè de Caen / Basse-Normandie, France, in 2005. From 2006 to 2014, he held an engineer position in Attitude and Orbit Control Subsystem within Astrium company, Airbus Group, in Toulouse. Since 2015, he has been an engineer within the Systems Biology group, MaIAGE, INRA, Université Paris-Saclay, in Jouy-en-Josas. His research control systems, particularly in biology.



Gérard Scorletti received the engineering degree from the École Nationale Supérieure de Techniques Avancées, Paris and his Ph.D. in Automatic Control from Orsay University, France in June 1997. He was a research associate at Centre for Systems Engineering Applied Mechanics, Belgium (1997-98). From 1998 to 2007, he was an assistant professor of Electrical Engineering at Université de Caen / BasseNormandie, France. Currently, he is a professor of Automatic control at École Centrale de Lyon, France, with a permanent joint appointment with the Ampère laboratory. His research interests include robust and LPV control, nonlinear control, identification for control and linear matrix inequality applications with a special emphasis on CAD.

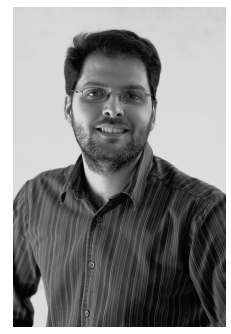

Florent Morel (S'06-M'07-SM'15) received the Aggregation in electrical engineering from the École Normale Superieure, Cachan, France, and the M.S. and $\mathrm{Ph} . \mathrm{D}$. degrees in electrical engineering from the Institut National de Sciences Appliquées, Lyon, France, in 2002, 2004, and 2007, respectively. His $\mathrm{Ph} . \mathrm{D}$. thesis dealt with predictive control, permanent magnet synchronous machines, voltage source inverters, and matrix converters. He is currently with the Ampère laboratory and the École Centrale, Lyon, France, as an Associate Professor. His research interests include power electronics and electromagnetic compatibility. 